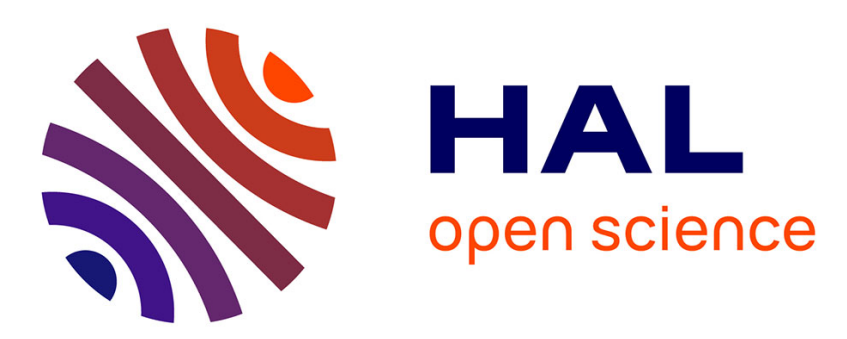

\title{
Discrete compactness for the hp version of rectangular edge finite elements
}

\author{
Daniele Boffi, Martin Costabel, Monique Dauge, Leszek Demkowicz
}

\section{To cite this version:}

Daniele Boffi, Martin Costabel, Monique Dauge, Leszek Demkowicz. Discrete compactness for the hp version of rectangular edge finite elements. SIAM Journal on Numerical Analysis, 2006, 44, pp.9791004. hal-00012191

\section{HAL Id: hal-00012191 https://hal.science/hal-00012191}

Submitted on 17 Oct 2005

HAL is a multi-disciplinary open access archive for the deposit and dissemination of scientific research documents, whether they are published or not. The documents may come from teaching and research institutions in France or abroad, or from public or private research centers.
L'archive ouverte pluridisciplinaire HAL, est destinée au dépôt et à la diffusion de documents scientifiques de niveau recherche, publiés ou non, émanant des établissements d'enseignement et de recherche français ou étrangers, des laboratoires publics ou privés. 


\title{
DISCRETE COMPACTNESS FOR THE $h p$ VERSION OF RECTANGULAR EDGE FINITE ELEMENTS
}

\author{
DANIELE BOFFI, MARTIN COSTABEL, MONIQUE DAUGE, AND LESZEK DEMKOWICZ
}

\begin{abstract}
Discretization of Maxwell eigenvalue problems with edge finite elements involves a simultaneous use of two discrete subspaces of $H^{1}$ and $\mathbf{H}$ (curl), reproducing the exact sequence condition. Kikuchi's Discrete Compactness Property, along with appropriate approximability conditions, implies the convergence of discrete eigenpairs to the exact ones.

In this paper we prove the discrete compactness property for the edge element approximation of Maxwell's eigenpairs on general $h p$ adaptive rectangular meshes. Hanging nodes, yielding 1-irregular meshes, are covered, and the order of the used elements can vary from one rectangle to another, thus allowing for a real $h p$ adaptivity. As a particular case, our analysis covers the convergence result for the $p$-method.
\end{abstract}

\section{INTRODUCTION}

The importance of the exact sequence

$$
H^{1} \stackrel{\text { grad }}{\longrightarrow} \mathbf{H}(\text { curl }) \stackrel{\text { curl }}{\longrightarrow} \mathbf{H}(\operatorname{div}) \stackrel{\text { div }}{\longrightarrow} L^{2}
$$

has been recognized in the analysis of Maxwell equations [12, 13, 2]. In two space dimensions the sequence reduces to

$$
H^{1} \stackrel{\text { grad }}{\longrightarrow} \mathbf{H}(\text { curl }) \stackrel{\text { curl }}{\longrightarrow} L^{2} .
$$

In this paper we shall deal with the two dimensional case.

The fundamental idea behind the construction of edge elements is based on the reproduction of the sequence at the discrete level. This idea had been also successfully exploited in the framework of mixed finite elements for elliptic problems, where it is also known as commuting diagram property [34].

Thus, we shall consider discrete subspaces of $H^{1}$ and $\mathbf{H}$ (curl) forming part of the discrete exact sequence. It is in this context that Kikuchi [35] introduced the fundamental notion of the Discrete Compactness Property which, along with appropriate approximability properties, guarantees the convergence of discrete Maxwell eigenvalues to the exact ones. We also

Date: 27 July 2005.

1991 Mathematics Subject Classification. 65N30, 65N25, 78M10.

Key words and phrases. Maxwell equations, $h p$ finite elements, discrete compactness, edge elements, eigenvalue approximation. 
refer to the book [17] for a definition corresponding to discrete compactness in an abstract setting. In another important contribution Caorsi et al. [16] have demonstrated that the discrete compactness property is not only a sufficient but also a necessary condition for the convergence of Maxwell eigenvalues without the appearance of any spurious mode.

Let us recall that the difficulty related with the Maxwell curlcurl operator is the lack of ellipticity manifested by the presence of the infinite dimensional kernel formed by the gradients. Nevertheless the Maxwell problem is well posed as soon as the divergence free constraint is imposed. With this constraint, the Maxwell problem recovers ellipticity properties due to the compact embedding of $\mathbf{H}_{0}$ (curl) $\cap \mathbf{H}$ (div) into $L^{2}$. The Discrete Compactness is the correct discrete analogue of the above compact embedding.

For the sake of clarity let us give the definition of Discrete Compactness Property. Like the usual approximability properties, it is related to a sequence of discrete spaces. Let $\left(\mathbf{X}_{n}\right)_{n}$ be a sequence of finite dimensional subspaces of $\mathbf{H}_{0}(\operatorname{curl})$ and $\left(\mathbb{Q}_{n}\right)_{n}$ a related sequence of subspaces of $H^{1}$. We say that the sequence $\left(\mathbb{Q}_{n}, \mathbf{X}_{n}\right)_{n}$ has the Discrete Compactness Property if there holds:

Any sequence $\mathbf{u}_{n} \in \mathbf{X}_{n}$ of discrete divergence free fields, i.e. satisfying

$$
\left(\mathbf{u}_{n}, \operatorname{grad} \phi_{n}\right)=0, \quad \forall \phi_{n} \in \mathbb{Q}_{n}
$$

and uniformly bounded in $\mathbf{H}$ (curl), has a subsequence converging in $L^{2}$.

The Discrete Compactness Property has been extensively studied in the framework of the $h$ version of edge finite elements where it is well known to hold true for a variety of edge finite elements on quite general two and three dimensional meshes (see the review papers [33, 19], the book [38], and the references therein, among which we recall in particular [35, 9, 6, 39, $7,16,14]$, but has not been widely investigated for the $p$ and $h p$ version yet. On the other hand, electromagnetic devices very often involve complicated geometries, which in particular may be neither smooth nor convex. The analysis of the singularities arising from reentrant corners or edges and from material discontinuities (see $[18,20]$ ) shows that such situations are to be handled with care. When using edge elements, one might want to locally adapt the meshsize $h$ and the approximation order $p$, which can possibly vary from one element to another within the same mesh. Such an hp strategy is an excellent way to get accurate results (an exponential convergence is expected and observed) when even severe singularities are present (see $[42,23]$ for examples of $h p$ finite element implementations).

In [8], the analysis of the discrete compactness property for triangular $h p$ finite elements has been tackled, but the proof of the main result relied on a conjectured $L^{2}$ estimate which had only been demonstrated numerically. Even for the pure $p$ method, there is no result in this direction available in the literature. In [37] the $p$ version of edge elements has been considered, but the proved results do not apply to eigenvalue approximations.

In this paper, we consider the two dimensional case of rectangular elements. A rigorous proof of the discrete compactness property is provided for edge elements of the first Nédélec 
family. Our hypotheses allow for a complete $h p$ refinement, including the presence of hanging nodes. The pure $p$ version of edge elements, being a subset of our setting, is naturally covered by our analysis. The same proof applies to meshes of quadrilaterals obtained by affine transformation from the reference square (i.e., parallelograms) and, more generally, to meshes obtained using the so called algebraic mesh generators.

The case of unstructured quadrilateral meshes presents some issues: It is known that the $h$ version of standard edge elements do not provide optimal results in this case, and, even, in the lowest order case there is no convergence at all, see [3]. Nevertheless, the results of [31] show that the Discrete Compactness Property holds in the framework of the $h$ version for the modified family of edge elements proposed and analyzed in [3]; another, simpler, modification has been proposed and analyzed in [11]. But the validity of the discrete compactness property for the $p$ and $h p$ versions of edge elements remains an open problem in the situation of unstructured meshes.

Our presentation starts with the pure $p$ method on a single square element, which is analyzed in Section 3 after the introduction of some preliminary notation in Section 2 . We consider, in particular, full tensor polynomials (the second Nédélec family of edge elements, see [41]) and standard edge element of the first Nédélec family (see [40]). We show that standard edge elements provide convergent approximation, while the second Nédélec family presents several spurious eigenpairs (more precisely, some discrete eigenvalues come with wrong multiplicity). We then present in Section 4 the general $h p$ theory which relies on an $L^{2}$ estimate which is proved thanks to the evaluation of an inf-sup constant on the reference element (Section 4.3). We make use of the $h p$ edge element spaces presented in [42], which generalize the first family of Nédélec finite elements.

We conclude our paper in Section 5, where we recall the consequences of the Discrete Compactness Property on the eigenvalue approximation by a Galerkin method: As a result, the $k$-th non-zero eigenvalue of the Galerkin discretization converges to the $k$-th non-zero Maxwell eigenvalue. We discuss the possibility of proving an exponential convergence rate, like obtained in [22] for the discretization of the Maxwell problem by the Weighted Regularization Method. We then finally comment on the extension of our proofs to the situation of general curvilinear polygons, with meshes obtained using algebraic mesh generators.

\section{Preliminary notions And notation}

2.1. Polynomial spaces on the reference square. The square is defined as $\Sigma:=I^{2}$ where $I$ is the interval $(-1,1)$. We denote the coordinates by $\mathbf{x}=(x, y)$. The outward unit normal vector on the boundary $\partial \Sigma$ is $\mathbf{n}$.

Everywhere $p$ denotes an integer $p \geq 1$. The space of polynomials of degree $\leq p$ on $I$ is denoted by $\mathbb{P}^{p}(I)$, its subspace of polynomials $\varphi$ with zero traces, $\varphi( \pm 1)=0$, is denoted by $\mathbb{P}_{0}^{p}(I)$. 
On the square, let $\mathbb{Q}^{p, q}(\Sigma)$ be the space of polynomials of separate degrees $p$ and $q$ in $x$ and $y$, respectively. This can be expressed as

$$
\mathbb{Q}^{p, q}(\Sigma)=\mathbb{P}^{p}(I) \otimes \mathbb{P}^{q}(I) .
$$

Symbol $\mathbb{Q}^{p}$ will be used for isotropic spaces, $\mathbb{Q}^{p}=\mathbb{Q}^{p, p}$, and $\mathbb{Q}_{0}^{p}$ will denote the polynomials with zero traces, $\mathbb{Q}_{0}^{p}=\mathbb{P}_{0}^{p} \otimes \mathbb{P}_{0}^{p}$.

We will study in the following two families of polynomial spaces for the electric field $\mathbf{u}=\left(u_{1}, u_{2}\right)$ on the square $\Sigma$. We postpone to Section 5 the introduction and the investigation of a third family, namely the augmented ABF family [3].

2.1.1. Full tensor product spaces: $\mathbf{Q}^{p}(\Sigma)$ denotes the full space $\mathbb{Q}^{p}(\Sigma) \times \mathbb{Q}^{p}(\Sigma)$. This is the space of Lagrange nodal elements on the square and, with appropriate degrees of freedom, this forms also the second Nédélec family of edge elements.

We denote by $\mathbf{Q}_{N}^{p}(\Sigma)$ its subspace of the fields $\mathbf{u}$ satisfying the perfect electric conductor boundary condition $\mathbf{u} \times \mathbf{n}=0$ on $\partial \Sigma$. We have

$$
\mathbf{Q}_{\mathrm{N}}^{p}(\Sigma)=\left[\mathbb{P}^{p}(I) \otimes \mathbb{P}_{0}^{p}(I)\right] \times\left[\mathbb{P}_{0}^{p}(I) \otimes \mathbb{P}^{p}(I)\right]
$$

2.1.2. Classical edge elements: $\mathbf{N}^{p}(\Sigma)$ denotes the space $\mathbb{Q}^{p-1, p}(\Sigma) \times \mathbb{Q}^{p, p-1}(\Sigma)$ which allows the commuting diagram property with the operator grad from the space of scalar polynomials $\mathbb{Q}^{p}(\Sigma)$. This edge element spaces are also known as first Nédélec family of edge elements. For simplicity, we shall refer to the spaces of this section as standard (Nédélec) edge elements. We denote by $\mathbf{N}_{N}^{p}(\Sigma)$ the subspace of fields satisfying the electric boundary condition:

$$
\mathbf{N}_{\mathrm{N}}^{p}(\Sigma)=\left[\mathbb{P}^{p-1}(I) \otimes \mathbb{P}_{0}^{p}(I)\right] \times\left[\mathbb{P}_{0}^{p}(I) \otimes \mathbb{P}^{p-1}(I)\right]
$$

2.2. Maxwell spectrum in the square. In Section 3 we shall describe in terms of 1D problems the Maxwell spectrum computed with the spaces $\mathbf{Q}_{\mathbf{N}}^{p}(\Sigma)$ and $\mathbf{N}_{\mathbf{N}}^{p}(\Sigma)$.

We first recall the definition of the standard continuous spaces associated with Maxwell equations on a domain $\Omega: \mathbf{H}(\operatorname{curl}, \Omega)$ is the space of $L^{2}(\Omega)$ fields with curl in $L^{2}(\Omega)$, while $\mathbf{H}_{0}(\operatorname{curl}, \Omega)$ is the subspace of $\mathbf{H}(\operatorname{curl}, \Omega)$ with perfect electric boundary conditions; $\mathbf{H}(\operatorname{div}, \Omega)$ is the space of $L^{2}(\Omega)$ fields with divergence in $L^{2}(\Omega)$.

Let us describe the Maxwell spectrum in the continuous space

$$
\mathbf{X}_{\mathrm{N}}(\Sigma):=\mathbf{H}_{0}(\operatorname{curl}, \Sigma) \cap \mathbf{H}(\operatorname{div}, \Sigma),
$$

i.e., the eigenpairs $(\lambda, \mathbf{u})$ with $\mathbf{u} \neq 0$ such that

$$
\mathbf{u} \in \mathbf{X}_{\mathrm{N}}(\Sigma): \quad \int_{\Sigma} \operatorname{curl} \mathbf{u} \operatorname{curl} \mathbf{v} d \mathbf{x}=\lambda \int_{\Sigma} \mathbf{u} \cdot \mathbf{v} d \mathbf{x}, \quad \forall \mathbf{v} \in \mathbf{X}_{\mathrm{N}}(\Sigma)
$$

2.2.1. The kernel: for $\lambda=0$, we have the whole space grad $H_{0}^{1}(\Sigma)$ of kernel elements. 
2.2.2. The genuine Maxwell spectrum: the whole non-zero spectrum corresponds to eigenvectors of the form $\mathbf{u}=\operatorname{curl} \varphi$ with $\varphi$ non-constant eigenvector of the Neumann problem on $\Sigma$. Let $\left(\psi_{j}\right)_{j \geq 0}$ be the basis of the Neumann eigenvectors on the interval $I$, they are associated with the eigenvalues $j^{2} \pi^{2} / 4$. Then, the Maxwell spectrum on $\Sigma$ is

$$
\lambda_{j, k}=\left(j^{2}+k^{2}\right) \pi^{2} / 4, \quad \mathbf{u}_{j, k}(x, y)=\left(\psi_{j}(x) \psi_{k}^{\prime}(y),-\psi_{j}^{\prime}(x) \psi_{k}(y)\right), \quad j+k \geq 1 .
$$

For comparison purposes, it is convenient to split the whole spectrum into the three following parts.

(a) The kernel.

(b) The non-zero Neumann eigenvalues $j^{2} \pi^{2} / 4$ associated with the two eigenvectors

$$
\left(0,-\psi_{j}^{\prime}(x)\right) \text { and }\left(\psi_{j}^{\prime}(y), 0\right) \text {. }
$$

(c) The sum of two non-zero Neumann eigenvalues $\left(j^{2}+k^{2}\right) \pi^{2} / 4$ with the eigenvectors $\mathbf{u}_{j, k}\left(\right.$ and $\mathbf{u}_{k, j}$ if $\left.j \neq k\right)$.

Remark 1. For all non-constant Neumann eigenvectors $\psi_{j}$ (i.e., for $j \geq 1$ ), $\psi_{j}^{\prime}$ is a Dirichlet eigenvector associated with the eigenvalue $j^{2} \pi^{2} / 4$. Let us denote $\varphi_{j}:=-\psi_{j}^{\prime}$. Then $\left(j^{2} \pi^{2} / 4\right) \psi_{j}=-\psi_{j}^{\prime \prime}=\varphi_{j}^{\prime}$, and we can see that eigenvectors associated with part (c) of the spectrum can be written as

$$
\left(\frac{1}{j^{2}} \varphi_{j}^{\prime}(x) \varphi_{k}(y),-\frac{1}{k^{2}} \varphi_{j}(x) \varphi_{k}^{\prime}(y)\right) .
$$

\section{Approximation of Maxwell's spectrum in a square by the $p$ method}

In this section we characterize explicitly the Maxwell spectrum on the square computed with the full polynomial space $\mathbf{Q}_{\mathrm{N}}^{p}(\Sigma)$ and with the Nédélec edge element space $\mathbf{N}_{\mathbf{N}}^{p}(\Sigma)$.

In contrast with (4), where the 1D generators are the Neumann eigenvectors on the interval, at the discrete level we will show that the 1D generators are the Dirichlet discrete eigenvectors: for $p \geq 2$, we consider the eigenpairs $(\lambda, \beta)$ with $\beta \neq 0$ such that

$$
\beta \in \mathbb{P}_{0}^{p}(I): \quad \int_{I} \beta^{\prime} w^{\prime} d x=\lambda \int_{I} \beta w d x, \quad \forall w \in \mathbb{P}_{0}^{p}(I) .
$$

The dimension of $\mathbb{P}_{0}^{p}(I)$ is $p-1$; for $j=1, \ldots, p-1$, let $\left(\lambda_{j}^{[p]}, \beta_{j}^{[p]}\right)$ be an eigenpair basis of (6) satisfying

$$
\lambda_{1}^{[p]}<\lambda_{2}^{[p]}<\ldots<\lambda_{p-1}^{[p]} .
$$

For each $j, \lambda_{j}^{[p]}$ tends exponentially to $j^{2} \pi^{2} / 4$ as $p \rightarrow \infty$. This follows from the standard convergence analysis for elliptic eigenvalue problems [5], and best approximation properties of the $p$-version of the FEM, see [43, Chapter 3]. 
We are going to describe the Maxwell spectrum in $\mathbf{Q}_{N}^{p}(\Sigma)$, i.e., the eigenpairs $(\lambda, \mathbf{u})$ such that

$$
\mathbf{u} \in \mathbf{Q}_{\mathrm{N}}^{p}(\Sigma): \quad \int_{\Sigma} \operatorname{curl} \mathbf{u} \operatorname{curl} \mathbf{v} d \mathbf{x}=\lambda \int_{\Sigma} \mathbf{u} \cdot \mathbf{v} d \mathbf{x}, \quad \forall \mathbf{v} \in \mathbf{Q}_{\mathrm{N}}^{p}(\Sigma)
$$

Theorem 1. The whole Maxwell spectrum $(7)$ in $\mathbf{Q}_{\mathrm{N}}^{p}(\Sigma)$ can be split into four parts.

(a) The kernel: $\lambda=0$ and $\mathbf{u} \in \operatorname{grad}\left(\mathbb{P}_{0}^{p} \otimes \mathbb{P}_{0}^{p}\right)$.

(b) The Dirichlet discrete eigenvalues $\lambda_{j}^{[p]}$ associated with the two eigenvectors

$$
\left(0,-\beta_{j}^{[p]}(x)\right) \quad \text { and } \quad\left(\beta_{j}^{[p]}(y), 0\right), \quad j=1, \ldots, p-1 .
$$

(c) The sum of two Dirichlet discrete eigenvalues $\lambda_{j}^{[p]}+\lambda_{k}^{[p]}$ with the eigenvectors

$$
\left(\lambda_{j}^{[p]} \beta_{k}^{[p] \prime}(x) \beta_{j}^{[p]}(y),-\lambda_{k}^{[p]} \beta_{k}^{[p]}(x) \beta_{j}^{[p] \prime}(y)\right), \quad 1 \leq j, k \leq p-1 .
$$

(d) The Dirichlet discrete eigenvalues $\lambda_{j}^{[p]}$ associated with two spurious eigenvectors

$$
\left(0,-\beta_{j}^{[p]}(x) L_{p}(y)\right) \quad \text { and } \quad\left(L_{p}(x) \beta_{j}^{[p]}(y), 0\right),
$$

where $L_{p}$ denotes the Legendre polynomial of degree $p$.

Remark 2. Note that formula (5) transforms into $\left(j^{2} \varphi_{k}^{\prime}(x) \varphi_{j}(y),-k^{2} \varphi_{k}(x) \varphi_{j}^{\prime}(y)\right)$ by swapping $j$ and $k$ and multiplying by $j^{2} k^{2}$. The similarity with formula (9) is now obvious.

Remark 3. The previous theorem shows that the space $\mathbf{Q}_{N}^{p}(\Sigma)$ is not suited for the computation of Maxwell's eigenvalues. Indeed, the discrete eigenvalues described in part (d) are redundant, providing a wrong multiplicity to the correct eigenvalues described in part (b). Moreover, the discrete eigenvectors of part (d) do not approximate any physical eigenfunction. The fact that the second Nédélec family produces spurious modes in the $h$-version of the FEM, has been documented in the literature, see e.g. [29, 30].

Proof. Let us first check the dimensions of the spaces described in the four above cases:
(a) $(p-1)^{2}$
(b) $2(p-1)$
(c) $(p-1)^{2}$
(d) $2(p-1)$.

The sum is $2(p-1)(p+1)$, which is the dimension of $\mathbf{Q}_{\mathbf{N}}^{p}(\Sigma)$ (see $\left.(1)\right)$.

It remains to check that the proposed pairs are eigenpairs of (7).

Case (a). The scalar polynomial space $\mathbb{P}_{0}^{p} \otimes \mathbb{P}_{0}^{p}$ is contained in $H_{0}^{1}(\Sigma)$, therefore all elements of $\operatorname{grad}\left(\mathbb{P}_{0}^{p} \otimes \mathbb{P}_{0}^{p}\right)$ belong to the kernel.

For the remaining part of the proof, since $p$ is fixed, let us drop the exponent $[p]$ in the notation of the discrete 1D Dirichlet eigenpairs. By integration by parts we note that the 
discrete eigenpairs $\left(\lambda_{j}, \beta_{j}\right)$ satisfy

$$
\int_{I}\left(\beta_{j}^{\prime \prime}+\lambda_{j} \beta_{j}\right) w d x=0, \quad \forall w \in \mathbb{P}_{0}^{p}(I)
$$

On the other hand, again by integration by parts, we obtain that $(\lambda, \mathbf{u})$ is an eigenpair in $\mathbf{Q}_{\mathrm{N}}^{p}(\Sigma)$, if and only if

$$
\mathbf{u} \in \mathbf{Q}_{\mathrm{N}}^{p}(\Sigma): \quad \int_{\Sigma}(\operatorname{curl} \operatorname{curl} \mathbf{u}-\lambda \mathbf{u}) \cdot \mathbf{v} d \mathbf{x}=0, \quad \forall \mathbf{v} \in \mathbf{Q}_{\mathrm{N}}^{p}(\Sigma) .
$$

It is clear that all proposed eigenvectors in (b), (c) and (d) belong to $\mathbf{Q}_{\mathrm{N}}^{p}(\Sigma)$. It remains to compute curl curl $\mathbf{u}-\lambda \mathbf{u}$ in each case and to check (12).

Case (b). For $\lambda=\lambda_{j}$ and $\mathbf{u}=\left(0,-\beta_{j}(x)\right)$, the two components of curl curl $\mathbf{u}-\lambda \mathbf{u}$ are

$$
0 \text { and } \beta_{j}^{\prime \prime}(x)+\lambda_{j} \beta_{j}(x) \text {. }
$$

Then relation (11) yields (12), and the same argument applies to the other vector $\left(\beta_{j}(y), 0\right)$.

Case (c). For $\lambda=\lambda_{j}+\lambda_{k}$ and $\mathbf{u}$ given by (9), we have

$$
\operatorname{curl} \mathbf{u}=\left(\lambda_{j}+\lambda_{k}\right) \beta_{k}^{\prime}(x) \beta_{j}^{\prime}(y)
$$

and the two components of $\operatorname{curl}$ curl $\mathbf{u}-\lambda \mathbf{u}$ are

$$
\begin{aligned}
& -\left(\lambda_{j}+\lambda_{k}\right) \beta_{k}^{\prime}(x) \beta_{j}^{\prime \prime}(y)-\left(\lambda_{j}+\lambda_{k}\right) \lambda_{j} \beta_{k}^{\prime}(x) \beta_{j}(y) \\
& +\left(\lambda_{j}+\lambda_{k}\right) \beta_{k}^{\prime \prime}(x) \beta_{j}^{\prime}(y)+\left(\lambda_{j}+\lambda_{k}\right) \lambda_{k} \beta_{k}(x) \beta_{j}^{\prime}(y)
\end{aligned}
$$

which can be written as

$$
\begin{gathered}
-\left(\lambda_{j}+\lambda_{k}\right) \beta_{k}^{\prime}(x)\left\{\beta_{j}^{\prime \prime}(y)+\lambda_{j} \beta_{j}(y)\right\} \\
\left(\lambda_{j}+\lambda_{k}\right) \beta_{j}^{\prime}(y)\left\{\beta_{k}^{\prime \prime}(x)+\lambda_{k} \beta_{k}(x)\right\} .
\end{gathered}
$$

Then relation (11) yields (12).

Case (d). For $\lambda=\lambda_{j}$ and $\mathbf{u}=\left(0,-\beta_{j}(x) L_{p}(y)\right)$, the two components of curl curl $\mathbf{u}-\lambda \mathbf{u}$ are

$$
-\beta_{j}^{\prime}(x) L_{p}^{\prime}(y) \quad \text { and } \quad \beta_{j}^{\prime \prime}(x) L_{p}(y)+\lambda_{j} \beta_{j}(x) L_{p}(y)
$$

The second component is orthogonal to any element of $\mathbb{P}_{0}^{p}(I) \otimes \mathbb{P}^{p}(I)$ (see (1) and (11)). It remains to check that the first component is orthogonal to $\mathbb{P}^{p}(I) \otimes \mathbb{P}_{0}^{p}(I)$, i.e.,

$$
\int_{\Sigma} v_{j}^{\prime}(x) L_{p}^{\prime}(y) w(x) v(y) d x d y=0, \quad \forall w \in \mathbb{P}^{p}(I), \quad \forall v \in \mathbb{P}_{0}^{p}(I) .
$$

It it sufficient to prove that $\int_{I} L_{p}^{\prime}(y) v(y) d y=0$ for all $v \in \mathbb{P}_{0}^{p}(I)$ : such a $v$ is given by $\left(1-y^{2}\right) \varphi(y)$ with $\varphi \in \mathbb{P}^{p-2}(I)$. Since the polynomials $L_{k}^{\prime}$ are orthogonal on $I$ with respect to the measure $\left(1-y^{2}\right) d y$ and since the degree of $L_{j}^{\prime}$ is $j-1$, we obtain that

$$
\int_{I} L_{p}^{\prime}(y) \varphi(y)\left(1-y^{2}\right) d y=0, \quad \forall \varphi \in \mathbb{P}^{p-2}(I)
$$


hence (13).

The next theorem characterizes the Maxwell spectrum in $\mathbf{N}_{\mathbf{N}}^{p}(\Sigma)$, i.e., the eigenpairs $(\lambda, \mathbf{u})$ such that

$$
\mathbf{u} \in \mathbf{N}_{\mathbf{N}}^{p}(\Sigma): \quad \int_{\Sigma} \operatorname{curl} \mathbf{u} \operatorname{curl} \mathbf{v} d \mathbf{x}=\lambda \int_{\Sigma} \mathbf{u} \cdot \mathbf{v} d \mathbf{x}, \quad \forall \mathbf{v} \in \mathbf{N}_{\mathbf{N}}^{p}(\Sigma)
$$

Theorem 2. The three first parts (a), (b), and (c) of the discrete spectrum described in Theorem 1 are the whole discrete Maxwell spectrum computed by the edge element space $\mathbf{N}_{\mathrm{N}}^{p}(\Sigma)$.

Proof. We can see that the eigenvectors of parts (a), (b), and (c) all belong to the smaller space $\mathbf{N}_{N}^{p}(\Sigma)$. Therefore they are also eigenvectors in this space. We see that the sum of the dimensions of the corresponding eigenspaces is $(p-1)^{2}+2(p-1)+(p-1)^{2}$, which is equal to $2(p-1) p$, the dimension of $\mathbf{N}_{\mathbf{N}}^{p}(\Sigma)$ (see $\left.(2)\right)$.

The conclusion arising from Theorems 1 and 2 is that the space $\mathbf{N}_{\mathbf{N}}^{p}(\Sigma)$ is to be preferred with respect to $\mathbf{Q}_{N}^{p}(\Sigma)$ for the computation of Maxwell's eigenpairs. Indeed, the latter space does not provide correct approximation of the spectrum (see Remark 3).

\section{Approximation of Maxwell's spectrum by $h p$ Rectangular finite elements}

In this section we extend the results about the space $\mathbf{N}_{N}^{p}(\Sigma)$ to the more involved situation of $h p$ refinements, which provides realistic applications to a class of polygonal domains. The structure of this section is as follows. First of all, we define the finite element spaces we are dealing with, and make precise the assumptions on the mesh. Then, after establishing an $L^{2}$ stability result (see Section 4.3), we prove the discrete compactness property which implies the convergence of eigenvalues/eigenvectors. Our proof clearly implies that the discrete compactness property holds true also for the pure $p$ method on a conforming rectangular mesh (i.e., without hanging node) with the standard edge elements of the first Nédélec family.

4.1. De Rham diagram for a variable order quad element. Let $\Sigma=I \times I$ be the master square element. A feature of edge elements is their embedding in a commuting de Rham diagram of type (15) relating two exact sequences of spaces, on both continuous and discrete levels. We refer to $[33,14]$ for a systematic description of the standard discrete de Rham diagram of any degree, where the interpolation operators are based on nodal values, edge and volume moments. In view of the construction $h p$ finite elements, another class of commuting de Rham diagram has been introduced [26], relying on the so called projectionbased interpolants, which allow variable orders on distinct elements of the same mesh, while preserving the commuting property. 
We start by introducing this latter version of the de Rham diagram, involving discrete spaces and interpolation operators on $\Sigma$, according to

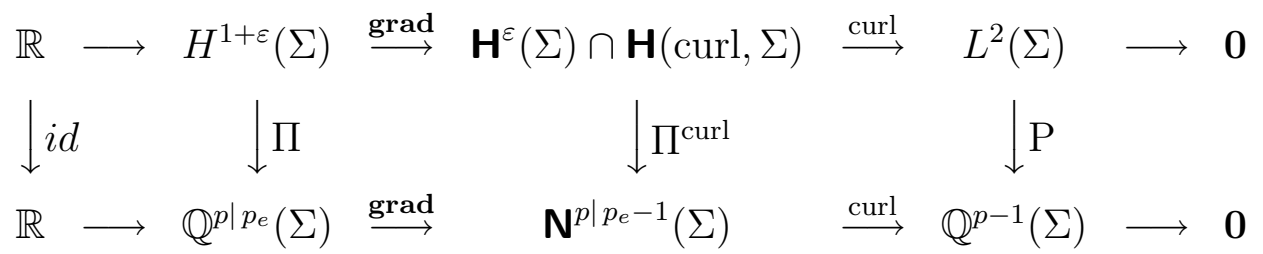

Index $p$ specifies the order in both variables which, for the sake of simplicity of this presentation, we assume to be identical, $\mathbb{Q}^{p}(\Sigma)=\mathbb{Q}^{p, p}(\Sigma)=\mathbb{P}^{p}(I) \otimes \mathbb{P}^{p}(I)$, and with every edge $e$ of the master element, we associate the corresponding order $p_{e}, e=1, \ldots, 4$ (with standard, counterclockwise enumeration of edges) that satisfies the condition:

$$
p_{e} \leq p, \quad e=1, \ldots, 4 .
$$

The polynomial spaces present in the diagram are defined as follows.

- $\mathbb{Q}^{p \mid p_{e}}(\Sigma)$ - the subspace of $\mathbb{Q}^{p}(\Sigma)$, consisting of polynomials whose traces on edges $e$ reduce to (possibly lower) order $p_{e}, e=1, \ldots, 4$.

- $\mathbf{N}^{p \mid p_{e}}(\Sigma)$ - the subspace of $\mathbf{N}^{p}(\Sigma)$, cf $\S 2.1 .2$, of vector-valued polynomials with traces of their tangential components on edges $e$ of (possibly lower) order $p_{e}$ :

$$
\mathbf{N}^{p \mid p_{e}}(\Sigma)=\left\{\mathbf{u} \in \mathbf{N}^{p}(\Sigma):\left.u_{t}\right|_{e}:=\left.(\mathbf{n} \times \mathbf{u})\right|_{e} \in \mathbb{P}^{p_{e}}(e), \forall e\right\},
$$

where $\mathbf{n}$ is the outward unit normal vector.

In particular, $\mathbb{Q}^{p \mid-1}$ provides an alternative notation for the subspace $\mathbb{Q}_{0}^{p}$ of polynomials vanishing on the boundary of the element, and $\mathbf{N}^{p \mid-1}=\mathbf{N}_{\mathbf{N}}^{p}$ stands for the subspace of vector-valued polynomials from the first Nédélec family whose tangential component traces on the boundary are equal to zero. The assumption that edge orders $p_{e}$ should not exceed corresponding components of order $p$, is realized in practice by implementing the minimum rule that sets an edge order $p_{e}$ to the minimum of orders $p$ corresponding to the adjacent elements.

4.1.1. $H^{1}$-conforming projection-based interpolation. Let $\mathbb{P}_{0}^{p}(I)$ denote the space of polynomials of degree $\leq p$, defined on the interval $I=(-1,1)$ with zero traces at the endpoints. Let $\phi_{1}(x)=(1-x) / 2, \phi_{2}(x)=(x+1) / 2$ be the standard 1D linear shape functions. Space $\mathbb{Q}^{p \mid} p_{e}(\Sigma)$ admits a natural decomposition into vertex bilinear shape functions, edge bubbles and element bubbles

$$
\begin{aligned}
\mathbb{Q}^{p \mid p_{e}}(\Sigma)= & \left\{\mathbb{P}^{1}(I) \otimes \mathbb{P}^{1}(I)\right\} \oplus \\
& \left\{\left(\mathbb{P}_{0}^{p_{1}}(I) \otimes \mathbb{R} \phi_{1}\right) \oplus\left(\mathbb{R} \phi_{2} \otimes \mathbb{P}_{0}^{p_{2}}(I)\right) \oplus\left(\mathbb{P}_{0}^{p_{3}}(I) \otimes \mathbb{R} \phi_{2}\right) \oplus\left(\mathbb{R} \phi_{1} \otimes \mathbb{P}_{0}^{p_{4}}(I)\right)\right\} \oplus \\
& \left\{\mathbb{P}_{0}^{p^{\prime}}(I) \otimes \mathbb{P}_{0}^{p}(I)\right\} .
\end{aligned}
$$


We will alternatively speak of edge bubbles for functions defined on a particular edge (and zero at its ends) or for their extensions to the whole element (and zero on the other edges). The linear extensions are natural but not essential in the forthcoming discussion. For a particular edge, the corresponding edge bubbles must vanish on the remaining edges and must "live" in the FE space. Similarly, the shape function for a vertex node must vanish at the remaining vertices and it must be in the FE space; the fact that it is constructed using bilinear functions, is secondary.

Given a function $u \in H^{1+\varepsilon}(\Sigma)$, we define its interpolant $u_{p}=\Pi u$, as a sum of three contributions,

$$
u_{p}=u_{1}+\underbrace{\sum_{e} u_{2, e, p}}_{u_{2, p}}+u_{3, p} .
$$

Interpolation at vertices. Vertex interpolant $u_{1}$ interpolates function $u$ at vertices,

$$
u_{1}(a)=u(a) \text { for each vertex } a \text {. }
$$

The simplest choice of an extension of the vertex values is provided by the bilinear function but the ultimate value of the interpolant is independent of the choice of the extension as long as the extension "lives" in the FE space.

Projection on edges. We subtract the vertex interpolant $u_{1}$ from $u$ and project the difference $u-u_{1}$, over each edge $e$, onto the space of edge bubbles,

$$
\left|u-u_{1}-u_{2, e, p}\right|_{1 / 2, e} \rightarrow \min .
$$

The projection is done in an $H^{1 / 2}(e)$ seminorm, and it is equivalent to the solution of a small linear system,

Find edge bubble $u_{2, e, p} \in \mathbb{P}_{0}^{p_{e}}(e)$ such that $\left(u-u_{1}-u_{2, e, p}, \phi\right)_{1 / 2, e}=0, \quad$ for each edge bubble $\phi \in \mathbb{P}_{0}^{p_{e}}(e)$,

where $(\cdot, \cdot)_{1 / 2, e}$ denotes the inner product corresponding to edge seminorm $|\cdot|_{1 / 2, e}$

Projection on the element. We extend each edge bubble $u_{2, e, p}$ to the whole element. Again, the most natural extension is provided by the element shape functions and corresponds to decomposition (19). We subtract then the total edge interpolant $u_{2, p}=\sum_{e} u_{2, e, p}$ from the difference $u-u_{1}$ and project the resulting difference on the element bubbles,

$$
\left|u-u_{1}-u_{2, p}-u_{3, p}\right|_{1, \Sigma} \rightarrow \min .
$$

Again, the projection is equivalent to a local Dirichlet problem on the element,

Find element bubble $u_{3, p} \in \mathbb{Q}_{0}^{p}(\Sigma)$ such that

$\left(u-u_{1}-u_{2, p}-u_{3, p}, \phi\right)_{1, \Sigma}=0, \quad$ for each element bubble $\phi \in \mathbb{Q}_{0}^{p}(\Sigma)$, 
where $(\cdot, \cdot)_{1, \Sigma}$ denotes the $H_{0}^{1}$ - inner product.

The interpolation is thus equivalent to the solution of a sequence of local (approximate) Dirichlet problems. We first interpolate at the vertices, and then, with the vertex values providing Dirichlet conditions, solve the edge Dirichlet problems. Finally, we use the vertex and edge interpolants to set up the Dirichlet boundary conditions and solve the final Dirichlet problem on the whole element. Remember that it does not matter in which way we construct lifts of the approximate Dirichlet data, the ultimate interpolant is unique. In each of the three steps, we determine a part of the interpolant corresponding to the decomposition (18).

4.1.2. $\mathbf{H}$ (curl)-conforming projection-based interpolation. A similar decomposition into edge functions and element bubbles can be constructed for the space $\mathbf{N}^{p \mid p_{e}-1}(\Sigma)$,

$$
\begin{aligned}
\mathbf{N}^{p \mid p_{e}-1}(\Sigma)=\{ & {\left[\left(\mathbb{P}^{p_{1}-1}(I) \otimes \mathbb{R} \phi_{1}\right) \times\{0\}\right] \oplus\left[\{0\} \times\left(\mathbb{R} \phi_{2} \otimes \mathbb{P}^{p_{2}-1}(I)\right)\right] \oplus } \\
& {\left.\left[\left(\mathbb{P}^{p_{3}-1}(I) \otimes \mathbb{R} \phi_{2}\right) \times\{0\}\right] \oplus\left[\{0\} \times\left(\mathbb{R} \phi_{1} \otimes \mathbb{P}^{p_{4}-1}(I)\right)\right]\right\} \oplus } \\
\{ & {\left.\left[\left(\mathbb{P}^{p-1} \otimes \mathbb{P}_{0}^{p}\right)(\Sigma) \times\{0\}\right] \oplus\left[\{0\} \times\left(\mathbb{P}_{0}^{p} \otimes \mathbb{P}^{p-1}\right)(\Sigma)\right]\right\} . }
\end{aligned}
$$

Given a vector-valued function $\mathbf{u} \in \mathbf{H}^{\varepsilon}(\operatorname{curl}, \Sigma)$, we define its interpolant $\mathbf{u}_{p}=\Pi^{\text {curl }} \mathbf{u}$ as a sum of two contributions,

$$
\mathbf{u}_{p}=\underbrace{\sum_{e} \mathbf{u}_{2, e, p}}_{\mathbf{u}_{2, p}}+\mathbf{u}_{3, p} .
$$

Edge projections. For each edge $e$, let $v_{t}=\mathbf{n} \times \mathbf{v}$ denote the (scalar-valued) tangential component $^{1}$ of a field $\mathbf{v}$ on $e$. We project the tangential component $u_{t}$ of function $\mathbf{u}$ onto the scalar edge functions,

$$
\left\|u_{t}-u_{2, e, p, t}\right\|_{-1 / 2, e} \rightarrow \min .
$$

Here the norm $\|\cdot\|_{-1 / 2, e}$ denotes the norm in the dual space

$$
H^{-1 / 2}(e)=\left(H_{00}^{1 / 2}(e)\right)^{\prime},
$$

see, e.g. [36]. We define then the vector edge function $\mathbf{u}_{2, e, p}$ as the tangent vector field on $e$ such that $u_{2, e, p, t}=\mathbf{n} \times \mathbf{u}_{2, e, p}$. The projection problem is equivalent to the variational problem

Find the tangential component $u_{2, e, p, t} \in \mathbb{P}^{p_{e}-1}(e)$ of the edge function $\mathbf{u}_{2, e, p}$ s.t.

$$
\left(u_{t}-u_{2, e, p, t}, \phi\right)_{-1 / 2, e}=0, \quad \text { for each edge function } \phi \in \mathbb{P}^{p_{e}-1}(e) \text {, }
$$

\footnotetext{
${ }^{1}$ More precisely, using the $3 \mathrm{D}$ notation, we have $v_{t}=(\mathbf{n} \times \mathbf{v}) \cdot \mathbf{e}_{z}$, where $\mathbf{e}_{z}$ is the unit vector orthogonal to the $2 \mathrm{D}$ plane.
} 
with $(\cdot, \cdot)_{-1 / 2, e}$ denoting the inner product corresponding to norm $\|\cdot\|_{-1 / 2, e}$. Notice that for a constant function $\phi$, the inner product reduces to $L^{2}$-product, and the equation above incorporates in particular the edge average condition

$$
\int_{e}\left(u_{t}-u_{2, e, p, t}\right) d s=0 .
$$

Element projection. We extend each individual edge function $\mathbf{u}_{2, e, p}$ to the whole element using the edge shape functions according to the splitting (20), sum it up, $\mathbf{u}_{2, p}=\sum_{e} \mathbf{u}_{2, e, p}$, and subtract the difference from function $\mathbf{u}$. We solve then a local projection problem,

$$
\left\|\operatorname{curl}\left(\mathbf{u}-\mathbf{u}_{2, p}-\mathbf{u}_{3, p}\right)\right\|_{0, \Sigma} \rightarrow \min
$$

subjected to the additional constraint,

$$
\left(\mathbf{u}-\mathbf{u}_{2, p}-\mathbf{u}_{3, p}, \operatorname{grad} \phi\right)=0, \quad \text { for each element scalar bubble } \phi .
$$

The constrained projection problem is equivalent to a Dirichlet mixed problem Find element bubble $\mathbf{u}_{3, p}$ and Lagrange multiplier $\psi$ such that

$$
\begin{cases}\left(\operatorname{curl}\left(\mathbf{u}-\mathbf{u}_{2, p}-\mathbf{u}_{3, p}\right), \operatorname{curl} \mathbf{v}\right)+(\operatorname{grad} \psi, \mathbf{v})=0 & \text { for every element vector bubble } \mathbf{v} \\ \left(\mathbf{u}-\mathbf{u}_{2, p}-\mathbf{u}_{3, p}, \operatorname{grad} \phi\right)=0 & \text { for every element scalar bubble } \phi .\end{cases}
$$

Here, the Lagrange multiplier $\psi$ lives in the space of scalar bubbles. Since grad $\psi$ is a vector bubble, the multiplier is identically equal zero and, for this reason, it is sometimes called the hidden variable.

Remark 4. In [24], the edge contributions $\mathbf{u}_{2, e, p}$ were split into the Whitney interpolant with constant tangential component, and a higher order edge bubble. Also, the choice of "edge" norms in the presentation above is consistent with the latest $3 \mathrm{D}$ results, see [25], and it is slightly different from those used in [24].

Finally $\mathrm{P}$ is the $L^{2}$ projection from $L^{2}(\Sigma)$ onto $\mathbb{Q}^{p-1}(\Sigma)$. With this, we have:

Theorem 3. If the edge seminorm $|u|_{1 / 2, e}$ is selected in such a way that

$$
|u|_{1 / 2, e}=\left\|\frac{\partial u}{\partial s}\right\|_{-1 / 2, e}
$$

then the de Rham diagram (15) commutes.

Proof. The tangential derivative $\partial_{s}$ is an isomorphism from $H^{1 / 2}(e) / \mathbb{R}$ onto $H^{-1 / 2}(e)$, see $\left[32\right.$, p. 31]). By the Bramble-Hilbert Lemma, the norm in the quotient space $H^{1 / 2}(e) / \mathbb{R}$ is

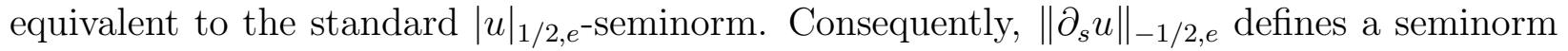
on $H^{1 / 2}(e)$, equivalent to the standard seminorm.

The commuting projection-based interpolation operators considered in [26] use different projections on edges based on $H^{1}$-seminorm for operator $\Pi$ and $L^{2}$-norm for operator $\Pi^{\text {curl }}$. 
The proof from [26] carries over to the case being presented without any modification, provided the $H^{1}$-seminorm is traded for the $H^{1 / 2}$-seminorm, and the $L^{2}$-norm is traded for $H^{-1 / 2}$-norm.

4.2. De Rham diagram for $h p$ meshes. In this section we consider a polygonal domain $\Omega$ with sides parallel to the axes, covered by rectangular meshes aligned along the same axes. Of course, by a global affine transformation, our result generalizes to the situation of non perpendicular axes.

If we fix a conforming mesh (i.e., such that the intersection of any two distinct elements $\bar{K}$ is either empty or a vertex or a full edge), and consider on each $K$ the mapped spaces $\mathbb{Q}^{p \mid p}(K), \mathbf{N}^{p \mid p-1}(K)$, and $\mathbb{Q}^{p-1}(K)$ with the same $p$, we can define on the whole domain $\Omega$ the corresponding $H^{1}-, \mathbf{H}(\operatorname{curl})$-, and $L^{2}$-conforming discrete spaces $Q_{p}, \mathbf{X}_{p}$ and $S_{p}$, and the projection-based interpolation is done element by element. The elements are said unconstrained in this case. Then it is clear that the commutativity properties (15) of the projection-based interpolation operators are still valid on the whole domain $\Omega$. Besides, we note that in this case the discrete spaces coincide with those of the standard $p$-extension of the edge elements [40,37].

The adaptation to $h p$ meshes containing local refinements, therefore hanging nodes, and variable degrees is by no means obvious.

For the sake of simplicity of the presentation, we shall restrict ourselves to 1-irregular $h p$ meshes corresponding to isotropic refinements only, and consisting of square elements. Beginning with a standard regular mesh consisting of square elements of the same size, we allow for breaking each element into four elements with the restriction that an element cannot share an edge with more than two small neighbors - the classical "two to one" rule. In other words, the generation level for two neighboring elements cannot differ by more than one. The order of elements can be modified locally, element by element, with the minimum rule being enforced - the order for an edge is set to the minimum of orders for all adjacent elements. Finally, since for meshes with hanging nodes the projections cannot be done on an element level - the resulting interpolants will no longer be conforming, the global conformity is maintained by means of the constrained approximation, see $[42,23]$.

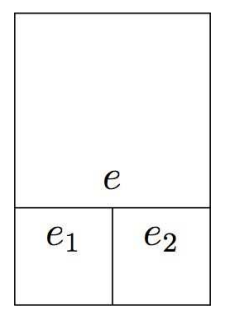

Figure 1. Constrained approximation 
The situation is illustrated in Fig.1. For a function $u$ defined on "big" edge $e$, the corresponding "big edge" interpolant is a polynomial defined on the whole edge, whereas the interpolants determined on the small edges $e_{1}, e_{2}$ result in a piecewise interpolant that, in general, is different.

A natural idea is to utilize the constrained approximation concepts. First do the projections on the big edges and, then, define the corresponding small edges interpolants by enforcing the global conformity requirements. The resulting interpolants will be indeed globally conforming, but we loose then the commutativity properties. This can be seen by considering the lowest order elements. The last space in the diagram reduces then to piecewise constants and the commutativity property requires that

$$
\int_{\partial K}\left(u_{p, t}-u_{t}\right) d s=\int_{K} \operatorname{curl}\left(\mathbf{u}_{p}-\mathbf{u}\right) d \mathbf{x}=0
$$

for each element $K$ in the mesh. For regular meshes, the condition follows from the edge averaging. In presence of hanging nodes, however, the condition may not be satisfied. Going back to the situation illustrated in Fig. 1, enforcing the averaging condition on "big" edge $e$ does not imply the same condition for the restrictions of the original function and its projection on the small edge $e_{1}$. Consequently, the condition above is violated for the small element, and the commutativity fails.

A remedy to the problem is to perform the interpolation on groups of elements. The whole mesh is split into polygonal patches consisting of single elements or element clusters (of minimum size) in such a way that all vertices of the polygonal patches are unconstrained. The decomposition is illustrated with the classical example of the L-shaped domain and $h$-refinements aimed at resolving the corner singularity shown in Fig 2.

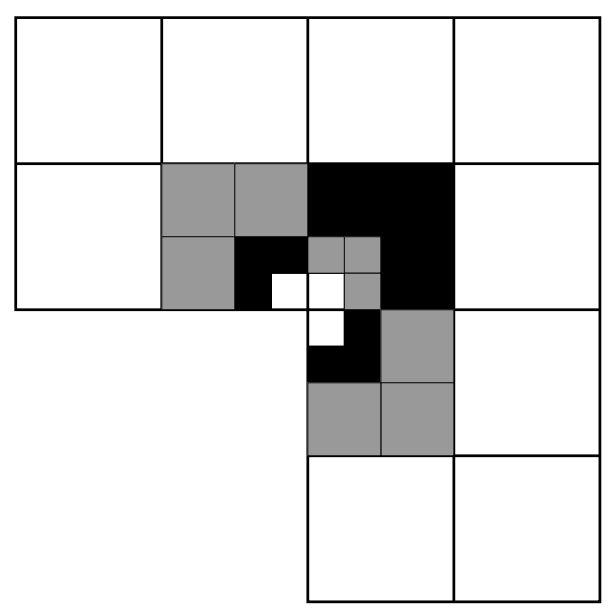

FiguRE 2. Decomposition of an 1-irregular mesh into clusters 
All clusters in this example coincide with either a single element (the white elements) or three elements forming an L-shaped patch (such clusters are indicated in the picture with a grey or black shading). In general, the 1-irregularity rule limits the number of possible cluster shapes to four cases only: clusters of a single, two, three or four small elements. Our convention is to call patch, denoted by $P$, the union of the cluster elements $K$ together with the interior edges. In our example, the L-shaped patches are the union of three squares and two interior edges, see Fig 3. Such patches have six distinct (exterior) edges. Edges of a patch always coincide with either a single element edge or two "small" edges adjacent to a big one.
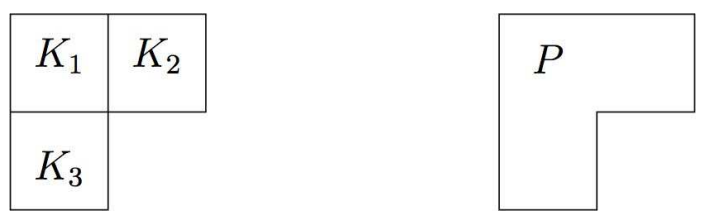

FiguRE 3. L-shaped cluster and patch

The space $\mathbb{Q}^{p \mid p_{e}}(P)$ on the patch is the subspace of continuous functions on $P$, which are $\mathbb{Q}^{p}$ on each element $K$ of the cluster, and whose restriction on $e$ belongs to $\mathbb{Q}^{p_{e}}(e)$ for each edge of the patch. The space $\mathbf{N}^{p \mid p_{e}-1}(P)$ is defined correspondingly as the subspace of $\mathbf{H}$ (curl) fields on $P$, which are in $\mathbf{N}^{p}$ on each element $K$, and whose tangential restriction on $e$ belongs to $\mathbb{Q}^{p_{e}}(e)$.

The definition of projection-based interpolation extends now naturally to the patch $P$ : We only list the main steps. The vertices are the corners of $P$ and we check that there exists a continuous piecewise bilinear vertex interpolant $u_{1} \in \mathbb{Q}^{1 / 1}(P)$. The edge bubbles $u_{2, e, p}$ are related with patch edges (and no more with element edges) and are polynomial on the whole patch edge. These bubbles can be extended inside $P$ as elements of $\mathbb{Q}^{p \mid p_{e}}(P)$. The patch bubbles $u_{3, p}$ are the functions in the FE space $\mathbb{Q}^{p \mid} p_{e}(P)$ with zero traces on the patch boundary $\partial P$.

Similarly we define the edge functions $\mathbf{u}_{2, e, p}$ as vector polynomials on the whole patch edge, tangential to the edge. They can be extended in $\mathbf{N}^{p \mid p_{e}-1}(P)$. The patch bubbles $\mathbf{u}_{3, p}$ are the elements of $\mathbb{Q}^{p \mid p_{e}}(P)$ with zero tangential traces on the patch boundary $\partial P$. The patch bubbles are, therefore, no longer polynomials but piecewise polynomials only.

Thus, by the same procedure as before we define the projection operators

$$
\Pi=\Pi_{P}, \quad \Pi^{\text {curl }}=\Pi_{P}^{\text {curl }} \quad \text { and } \quad \mathrm{P}=\mathrm{P}_{P}
$$

and obtain a commutative scheme like (15). 
Once the interpolation is done on the patches, we regain both the global conformity and commutativity of the interpolation operators on the whole $h p$ mesh:

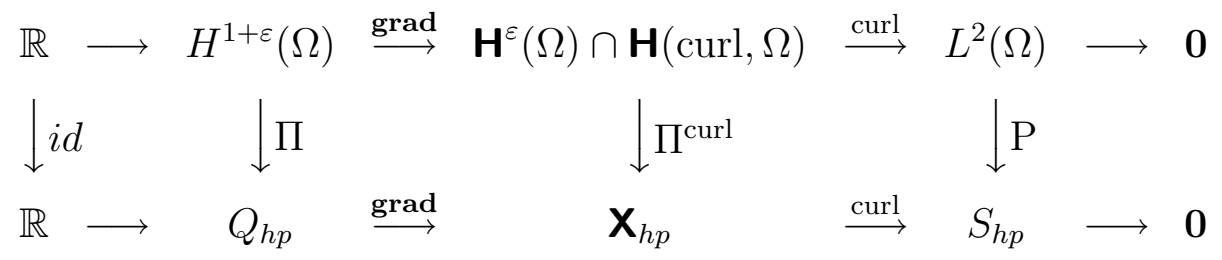

Here $Q_{h p}, \mathbf{X}_{h p}, S_{h p}$ denote FE spaces defined on the common domain $\Omega$, corresponding to the $H^{1}-, \mathbf{H}$ (curl)-, and $L^{2}$-conforming discretizations, done patch by patch.

4.3. A stability result in $L^{2}$. We begin by recalling the inclusion of polynomial spaces,

$$
\mathbb{Q}_{0}^{p}(\Sigma) \stackrel{\operatorname{grad}}{\longrightarrow} \mathbf{N}_{\mathrm{N}}^{p}(\Sigma)
$$

Here,

$$
\begin{aligned}
\mathbb{Q}_{0}^{p}(\Sigma) & =\mathbb{P}_{0}^{p}(I) \otimes \mathbb{P}_{0}^{p}(I) \\
\mathbf{N}_{\mathrm{N}}^{p}(\Sigma) & =\left[\mathbb{P}^{p-1}(I) \otimes \mathbb{P}_{0}^{p}(I)\right] \times\left[\mathbb{P}_{0}^{p}(I) \otimes \mathbb{P}^{p-1}(I)\right] .
\end{aligned}
$$

In this section, we will omit the mention of $\Sigma$ and $I$ for the spaces $\mathbb{Q}^{p}, \mathbf{N}^{p}$, and $\mathbb{P} p$, respectively. We shall denote the $L^{2}$-norm on $I$ or $\Sigma$, by $\|\cdot\|$, with the corresponding $L^{2}$-product denoted by $(\cdot, \cdot)$. We hope that the similarity of the latter with the notation for vector components will not lead to confusion.

Theorem 4. The following stability condition holds:

$$
\inf _{\mathbf{q} \in \mathbf{N}_{\mathbf{N}}^{p}} \sup _{\mathbf{s} \in \operatorname{grad} \mathbb{Q}_{0}^{p} \oplus \mathbf{c u r l} \operatorname{curl} \mathbf{N}_{\mathrm{N}}^{p}} \frac{(\mathbf{q}, \mathbf{s})}{\|\mathbf{q}\|\|\mathbf{s}\|}=C_{p},
$$

where

$$
C_{p}=\left(\frac{2(2 p+1)}{(p+1)(p+2)}\right)^{1 / 2}=O\left(p^{-1 / 2}\right)
$$

The proof of Theorem 4 relies on two lemmas.

Lemma 5. Let $a_{i}>0, b_{i}>0, i=1, \ldots, n$. Then for any real $v_{1}, \ldots, v_{n}$

$$
\sup _{u_{1}, \ldots, u_{n}} \frac{\left|\sum_{i=1}^{n} a_{i} u_{i} v_{i}\right|}{\left(\sum_{i=1}^{n} b_{i} u_{i}^{2}\right)^{1 / 2}}=\left(\sum_{i=1}^{n} \frac{a_{i}^{2}}{b_{i}} v_{i}^{2}\right)^{1 / 2} .
$$


Proof. Use Cauchy-Schwarz inequality for the discrete $l^{2}$ product and the representation,

$$
\sum_{i=1}^{n} a_{i} u_{i} v_{i}=\sum_{i=1}^{n} \frac{a_{i}}{b_{i}^{1 / 2}} v_{i} b_{i}^{1 / 2} u_{i}
$$

We recall that $\left(\lambda_{i}, \beta_{i}\right), i=1, \ldots, p-1$, denote the discrete eigenpairs of the $1 \mathrm{D}$ Laplace operator defined in equation (6) (we omit the exponent $[p]$ for simplicity). The eigenvectors are normalized to satisfy $\left(\beta_{i}, \beta_{j}\right)=\delta_{i j}$.

Lemma 6. The following inequality holds.

$$
\left(\sum_{i=1}^{p-1} \lambda_{i}^{2} v_{i}^{2}\right)^{1 / 2} \geq C_{p}\left\|\sum_{i=1}^{p-1} v_{i} \beta_{i}^{\prime \prime}\right\|, \quad \forall \mathbf{v}=\left(v_{1}, \ldots, v_{p-1}\right) \in \mathbb{R}^{p-1},
$$

where $C_{p}$ is defined in (24).

Proof. It was proved in [8] that the constant

$$
\begin{aligned}
C_{p} & =\inf _{u \in \mathbb{P}_{0}^{p}} \sup _{f \in \mathbb{P}^{p-2}} \frac{(u, f)}{\|u\|\|f\|}=\inf _{u \in \mathbb{P}_{0}^{p}} \sup _{v \in \mathbb{P}_{0}^{p}} \frac{\left(u, v^{\prime \prime}\right)}{\|u\|\left\|v^{\prime \prime}\right\|} \\
& =\inf _{u \in \mathbb{P}_{0}^{p}} \sup _{v \in \mathbb{P}_{0}^{p}} \frac{\left(u^{\prime}, v^{\prime}\right)}{\|u\|\left\|v^{\prime \prime}\right\|}=\inf _{v \in \mathbb{P}_{0}^{p}} \sup _{u \in \mathbb{P}_{0}^{p}} \frac{\left(u^{\prime}, v^{\prime}\right)}{\|u\|\left\|v^{\prime \prime}\right\|}
\end{aligned}
$$

is given by formula (24). Consequently,

$$
\sup _{u \in \mathbb{P}_{0}^{p}} \frac{\left(u^{\prime}, v^{\prime}\right)}{\|u\|} \geq C_{p}\left\|v^{\prime \prime}\right\|, \quad \forall v \in \mathbb{P}_{0}^{p} .
$$

If we now define

$$
u=\sum_{i=1}^{p-1} u_{i} \beta_{i}, \quad v=\sum_{i=1}^{p-1} v_{i} \beta_{i}
$$

then

$$
\left(u^{\prime}, v^{\prime}\right)=\sum_{i=1}^{p-1} \lambda_{i} u_{i} v_{i} \quad \text { and } \quad\|u\|=\left(\sum_{i=1}^{p-1} u_{i}^{2}\right)^{1 / 2} .
$$

Apply Lemma 5 to finish the proof.

Proof of Theorem 4.

Step 1: let $\alpha_{i}, i=0, \ldots, p-1$ be a basis for $\mathbb{P}^{p-1}$ defined as follows

$$
\alpha_{i}= \begin{cases}1 / \sqrt{2} & i=0 \\ \beta_{i}^{\prime} & i=1, \ldots, p-1 .\end{cases}
$$


Polynomials $\alpha_{i}$ are orthogonal and satisfy

$$
\left\|\alpha_{0}\right\|^{2}=1, \quad\left\|\alpha_{i}\right\|^{2}=\lambda_{i}, i=1, \ldots, p-1 .
$$

Any element $\mathbf{q} \in \mathbf{N}_{\mathrm{N}}^{p}$ can be represented in the form,

$$
\mathbf{q}=\left(\sum_{i=0}^{p-1} \sum_{j=1}^{p-1} q_{1, i j} \alpha_{i} \beta_{j}, \sum_{i=1}^{p-1} \sum_{j=0}^{p-1} q_{2, i j} \beta_{i} \alpha_{j}\right) .
$$

Here and in what follows, we assume that in a tensor product $\alpha \beta$, the first function is always a function of $x$, and the second is a function of $y$, i.e., $\alpha \beta=\alpha(x) \beta(y)$.

A direct calculation shows that,

$$
\begin{gathered}
\text { curl curl } \mathbf{q}=\left(\sum_{i=1}^{p-1} \sum_{j=0}^{p-1} q_{2, i j} \beta_{i}^{\prime} \alpha_{j}^{\prime}-\sum_{i=0}^{p-1} \sum_{j=1}^{p-1} q_{1, i j} \alpha_{i} \beta_{j}^{\prime \prime},-\sum_{i=1}^{p-1} \sum_{j=0}^{p-1} q_{2, i j} \beta_{i}^{\prime \prime} \alpha_{j}+\sum_{i=0}^{p-1} \sum_{j=1}^{p-1} q_{1, i j} \alpha_{i}^{\prime} \beta_{j}^{\prime}\right) \\
=\left(\sum_{i=1}^{p-1} \sum_{j=1}^{p-1} q_{2, i j} \beta_{i}^{\prime} \beta_{j}^{\prime \prime}-\sum_{i=0}^{p-1} \sum_{j=1}^{p-1} q_{1, i j} \alpha_{i} \beta_{j}^{\prime \prime},-\sum_{i=1}^{p-1} \sum_{j=0}^{p-1} q_{2, i j} \beta_{i}^{\prime \prime} \alpha_{j}+\sum_{i=1}^{p-1} \sum_{j=1}^{p-1} q_{1, i j} \beta_{i}^{\prime \prime} \beta_{j}^{\prime}\right) \\
=\left(-\sum_{j=1}^{p-1} q_{1,0 j} \alpha_{0} \beta_{j}^{\prime \prime}+\sum_{i=1}^{p-1} \sum_{j=1}^{p-1}\left(q_{2, i j}-q_{1, i j}\right) \beta_{i}^{\prime} \beta_{j}^{\prime \prime},-\sum_{i=1}^{p-1} q_{2, i 0} \beta_{i}^{\prime \prime} \alpha_{0}-\sum_{i=1}^{p-1} \sum_{j=1}^{p-1}\left(q_{2, i j}-q_{1, i j}\right) \beta_{i}^{\prime \prime} \beta_{j}^{\prime}\right) .
\end{gathered}
$$

Hence, any element $\mathbf{s} \in \operatorname{curl}$ curl $\mathbf{N}_{\mathrm{N}}^{p}$ can be represented in the form,

$$
\mathbf{s}=\left(\sum_{j=1}^{p-1} s_{0 j} \alpha_{0} \beta_{j}^{\prime \prime}+\sum_{i=1}^{p-1} \sum_{j=1}^{p-1} s_{i j} \beta_{i}^{\prime} \beta_{j}^{\prime \prime}, \sum_{i=1}^{p-1} s_{i 0} \beta_{i}^{\prime \prime} \alpha_{0}-\sum_{i=1}^{p-1} \sum_{j=1}^{p-1} s_{i j} \beta_{i}^{\prime \prime} \beta_{j}^{\prime}\right) .
$$

Let $\mathbf{q} \in \mathbf{N}_{\mathrm{N}}^{p}$ be discrete divergence free, i.e.,

$$
(\mathbf{q}, \operatorname{grad} w)=0, \quad \forall w \in \mathbb{Q}_{0}^{p} .
$$

Selecting $w=\beta_{k} \beta_{l}, k, l=1, \ldots, p-1$, we conclude that coefficients $q_{1, i j}, q_{2, i j}$ in representation (25) must satisfy the identity

$$
q_{1, k l} \lambda_{k}+q_{2, k l} \lambda_{l}=0 .
$$

This leads to the following formulas for the norm of a discrete divergence free vector, and the $L^{2}$-product of such a vector with $\mathbf{s} \in \operatorname{curl} \operatorname{curl} \mathbf{N}_{\mathrm{N}}^{p}$

$$
\begin{gathered}
\|\mathbf{q}\|^{2}=\sum_{j=1}^{p-1} q_{1,0 j}^{2}+\sum_{i=1}^{p-1} q_{2, i 0}^{2}+\sum_{i=1}^{p-1} \sum_{j=1}^{p-1}\left(\frac{\lambda_{i}^{2}}{\lambda_{j}}+\lambda_{i}\right) q_{1, i j}^{2} \\
(\mathbf{q}, \mathbf{s})=-\sum_{j=1}^{p-1} q_{1,0 j} s_{0 j} \lambda_{j}-\sum_{i=1}^{p-1} q_{2, i 0} s_{i 0} \lambda_{i}-\sum_{i=1}^{p-1} \sum_{j=1}^{p-1} q_{1, i j} s_{i j}\left(\lambda_{i} \lambda_{j}+\lambda_{i}^{2}\right) .
\end{gathered}
$$


Applying Lemma 5 we get,

$$
\sup _{\substack{\mathbf{q} \in \mathbf{N}_{N}^{p} \\(\mathbf{q}, \operatorname{grad} w)=0, \forall w \in \mathbb{Q}_{0}^{p}}} \frac{(\mathbf{q}, \mathbf{s})}{\|\mathbf{q}\|}=\sum_{j=1}^{p-1} \lambda_{j}^{2} s_{0 j}^{2}+\sum_{i=1}^{p-1} \lambda_{i}^{2} s_{i 0}^{2}+\sum_{i=1}^{p-1} \sum_{j=1}^{p-1}\left(\lambda_{i}^{2} \lambda_{j}+\lambda_{i} \lambda_{j}^{2}\right) s_{i j}^{2} .
$$

Finally, the norm of $\mathbf{s} \in \mathbf{c u r l} \operatorname{curl} \mathbf{N}_{\mathrm{N}}^{p}$ can be represented in the form,

$$
\|\mathbf{s}\|^{2}=\left\|\sum_{j=1}^{p-1} s_{0 j} \beta_{j}^{\prime \prime}\right\|^{2}+\left\|\sum_{i=1}^{p-1} s_{i 0} \beta_{i}^{\prime \prime}\right\|^{2}+\sum_{i=1}^{p-1} \lambda_{i}\left\|\sum_{j=1}^{p-1} s_{i j} \beta_{j}^{\prime \prime}\right\|^{2}+\sum_{j=1}^{p-1} \lambda_{j}\left\|\sum_{i=1}^{p-1} s_{i j} \beta_{i}^{\prime \prime}\right\|^{2} .
$$

By Lemma 6 we have,

$$
\begin{gathered}
\sum_{j=1}^{p-1} \lambda_{j}^{2} s_{0 j}^{2} \geq C_{p}^{2}\left\|\sum_{j=1}^{p-1} s_{0 j} \beta_{j}^{\prime \prime}\right\|^{2} \\
\sum_{i=1}^{p-1} \lambda_{i}^{2} s_{i 0}^{2} \geq C_{p}^{2}\left\|\sum_{i=1}^{p-1} s_{i 0} \beta_{i}^{\prime \prime}\right\|^{2} \\
\lambda_{i} \sum_{j=1}^{p-1} \lambda_{j}^{2} s_{i j}^{2} \geq \lambda_{i} C_{p}^{2}\left\|\sum_{j=1}^{p-1} s_{i j} \beta_{j}^{\prime \prime}\right\|^{2} \\
\lambda_{j} \sum_{i=1}^{p-1} \lambda_{i}^{2} s_{i j}^{2} \geq \lambda_{j} C_{p}^{2}\left\|\sum_{i=1}^{p-1} s_{i j} \beta_{i}^{\prime \prime}\right\|^{2} .
\end{gathered}
$$

Summing up all the inequalities, we get,

$$
\sup _{\substack{\mathbf{q} \in \mathbf{N}_{\mathrm{N}}^{p} \\(\mathbf{q}, \operatorname{grad} w)=0, \forall w \in \mathbb{Q}_{0}^{p}}} \frac{(\mathbf{q}, \mathbf{s})}{\|\mathbf{q}\|} \geq C_{p}\|\mathbf{s}\|,
$$

or, equivalently, using the equality of inf-sup constants for a bilinear form and its adjoint,

$$
\inf _{\substack{\mathbf{q} \in \mathbf{N}_{\mathrm{N}}^{p} \\(\mathbf{q}, \operatorname{grad} w)=0, \forall w \in \mathbb{Q}_{0}^{p}}} \sup _{\mathbf{s} \in \mathbf{c u r l} \operatorname{curl} \mathbf{N}_{\mathrm{N}}^{p}} \frac{(\mathbf{q}, \mathbf{s})}{\|\mathbf{q}\|\|\mathbf{s}\|} \geq C_{p} .
$$

Step 2: use discrete Helmholtz decomposition,

$$
\mathbf{q}=\mathbf{q}_{0}+\operatorname{grad} \phi, \quad\left(\mathbf{q}_{0}, \operatorname{grad} w\right)=0 \forall w \in \mathbb{Q}_{0}^{p}, \quad \phi \in \mathbb{Q}_{0}^{p} .
$$

to extend inequality $(26)$ to arbitrary $\mathbf{q} \in \mathbf{N}_{\mathbf{N}}^{p}$ and $\mathbf{s} \in \operatorname{grad} \mathbb{Q}_{0}^{p} \oplus \operatorname{curl} \operatorname{curl} \mathbf{N}_{\mathrm{N}}^{p}$. 
Step 3: The equality in (23) follows, e.g., from the fact that for q coinciding with eigenvectors from part (b) of the spectrum (see (8)), the two-dimensional inf-sup condition reduces to its one-dimensional counterpart.

The consequence of Theorem 4 is the following $L^{2}$ stability result in $p$-version:

Theorem 7. Let $\mathbf{u}_{3} \in \mathbf{H}^{\varepsilon}(\Sigma) \cap \mathbf{H}_{0}(\operatorname{curl}, \Sigma)$ be a divergence free bubble function on $\Sigma$. Let $\mathbf{u}_{3, p}$ be the projection $\Pi^{\text {curl }} \mathbf{u}_{3}$. Then $\mathbf{u}_{3, p}$ is discrete divergence free and there holds

$$
\left\|\mathbf{u}_{3}-\mathbf{u}_{3, p}\right\|_{0, \Sigma} \leq C p^{1 / 2} \inf _{\mathbf{q}_{p} \in \mathbf{N}_{\mathrm{N}}^{p}}\left\|\mathbf{u}_{3}-\mathbf{q}_{p}\right\|_{0, \Sigma}
$$

Proof. Let $\mathbf{q}_{p}$ be any element of $\mathbf{N}_{\mathrm{N}}^{p}$. Since

$$
\left(\Pi^{\text {curl }} \mathbf{u}_{3}, \operatorname{grad} \mathbf{q}_{p}\right)=\left(\mathbf{u}_{3}, \Pi^{\text {curl }} \operatorname{grad} \mathbf{q}_{p}\right)=\left(\mathbf{u}_{3}, \operatorname{grad} \Pi \mathbf{q}_{p}\right),
$$

we obtain that $\mathbf{u}_{3, p}$ is discrete divergence-free.

By Theorem 4, there exists $\mathbf{s} \in \operatorname{grad} \mathbb{Q}_{0}^{p} \oplus \operatorname{curl} \operatorname{curl} \mathbf{N}_{\mathrm{N}}^{p}$ so that

$$
C_{p}\left\|\mathbf{u}_{3, p}-\mathbf{q}_{p}\right\|\|\mathbf{s}\| \leq\left(\mathbf{u}_{3, p}-\mathbf{q}_{p}, \mathbf{s}\right) .
$$

Any $\mathbf{s} \in \operatorname{grad} \mathbb{Q}_{0}^{p} \oplus \operatorname{curl} \operatorname{curl} \mathbf{N}_{\mathrm{N}}^{p}$ being orthogonal to $\mathbf{u}_{3}-\mathbf{u}_{3, p}$ we get

$$
C_{p}\left\|\mathbf{u}_{3, p}-\mathbf{q}_{p}\right\|\|\mathbf{s}\| \leq\left(\mathbf{u}_{3}-\mathbf{q}_{p}, \mathbf{s}\right) \leq\left\|\mathbf{u}_{3}-\mathbf{q}_{p}\right\|\|\mathbf{s}\| .
$$

By the triangle inequality we deduce (27).

The best approximation error in the $L^{2}$ norm by polynomials in $\mathbf{N}_{\mathrm{N}}^{p}$ behaves as $p^{-1}$ for fields in $H^{1}$ satisfying the boundary conditions of $\mathbf{H}_{0}$ (curl):

Lemma 8. Let $\mathbf{u}_{3} \in \mathbf{H}^{1}(\Sigma) \cap \mathbf{H}_{0}$ (curl, $\Sigma$ ) be a general bubble function on $\Sigma$. There exists $\mathbf{q}_{p} \in \mathbf{N}_{\mathbf{N}}^{p}(\Sigma)$ such that

$$
\left\|\mathbf{u}_{3}-\mathbf{q}_{p}\right\|_{0, \Sigma} \leq C p^{-1}\left\|\mathbf{u}_{3}\right\|_{1, \Sigma}
$$

Proof. Let $u_{x}$ and $u_{y}$ be the two components of $\mathbf{u}_{3}$. We note that $u_{x}$ belongs to $L^{2}\left(I, H_{0}^{1}(I)\right) \cap$ $H^{1}\left(I, L^{2}(I)\right)$. We take as interpolant for $u_{x}$ the function $\pi_{x}^{p-1,0} \otimes \pi_{y}^{p, 1}\left(u_{x}\right)$ where $\pi^{p, 0}$ and $\pi^{p, 1}$ are the $1 \mathrm{D}$ standard projection operators used in spectral and $p$ methods: $\pi^{p, 0}$ is the $L^{2}$ orthogonal projection on $\mathbb{P}^{p}(I)$ and $\pi^{p, 1}$ is defined as

$$
\pi^{p, 1}(u)(t)=\int_{-1}^{t} \pi^{p-1,0}\left(u^{\prime}\right)(s) d s .
$$

Both $\pi^{p, 0}$ and $\pi^{p, 1}$ satisfy the $L^{2}-H^{1}$ error estimate with a factor $p^{-1}$, see [43, Ch.3] for instance. Moreover $\pi^{p, 0}$ is stable in $L^{2}$ and $\pi^{p, 1}$ in $H^{1}$. The proof of the estimate for $\left\|u_{x}-\pi_{x}^{p-1,0} \otimes \pi_{y}^{p, 1}\left(u_{x}\right)\right\|_{0, \Sigma}$ then follows. The situation for the second component is similar. 
4.4. Discrete compactness. In this section we prove the discrete compactness property for edge finite elements on 1-irregular $h p$ square meshes. The discrete compactness property, stated in Theorem 11, is known to be sufficient and in a sense necessary for the good approximation of eigenvalues/eigenvectors (see [33, 7, 16, 39], for instance).

For our proof we need $L^{2}$ estimates for $\mathbf{u}-\Pi^{\text {curl }} \mathbf{u}$ for divergence free fields $\mathbf{u}$ on any unconstrained element $K$ (Lemma 9) or any patch $P$ (Lemma 10).

Lemma 9. Let $K$ be an unconstrained square element of size $h=h_{K}$, and let $p$ be the minimum among $p_{K}$ and $\left\{p_{e}, e=1, \ldots, 4\right\}$. Let $\mathbf{u} \in \mathbf{H}^{r}(K), 0<r<1 / 2$, curl $\mathbf{u} \in L^{2}(K)$, $\operatorname{div} \mathbf{u}=0$. For every $\varepsilon>0$, there exists a constant $C>0$, dependent upon $\varepsilon$ but independent of the element and function $\mathbf{u}$, such that

$$
\left\|\mathbf{u}-\Pi_{K}^{\text {curl }} \mathbf{u}\right\| \leq C\left(\frac{h}{p}\right)^{(r-\varepsilon)}\left(\|\mathbf{u}\|_{r, K}+\|\operatorname{curl} \mathbf{u}\|_{0, K}\right) .
$$

Here $\Pi_{K}^{\text {curl }}$ is the projection-based interpolation on $K$ transported from $\Pi^{\text {curl }}$ in (15).

Proof. Step 1: $p$-estimate on the master element. Assume first that $K=\Sigma$ is the master square element. It follows from the integration by parts formula

$$
\int_{K}(\operatorname{curl} \mathbf{u}) \phi d \mathbf{x}=\int_{K} \mathbf{u} \cdot \operatorname{curl} \phi d \mathbf{x}+\int_{\partial K} u_{t} \phi d s
$$

that the tangential component $u_{t}$ lives in $H^{-1 / 2+r}(\partial K)$ and

$$
\left\|u_{t}\right\|_{-1 / 2+r, \partial K} \leq C\left(\|\mathbf{u}\|_{r, K}+\|\operatorname{curl} \mathbf{u}\|_{0, K}\right),
$$

with $C$ denoting a generic constant depending upon the master element only. We decompose function $\mathbf{u}$ into three contributions

$$
\mathbf{u}=\mathbf{u}_{1}+\operatorname{grad} q+\mathbf{u}_{3} .
$$

The terms are constructed as follows.

- $\mathbf{u}_{1}$ is the lowest degree Whitney interpolant, which means that $\mathbf{u}_{1} \in \mathbf{N}^{1 \mid 0}(K)$, div $\mathbf{u}_{1}=$ 0 and the tangential traces of $\mathbf{u}_{1}$ are the mean values of those of $\mathbf{u}$ on each edge of $K$.

- Potential $q$ is obtained by integrating tangential component $u_{t}-u_{1 t}$ along the element boundary, starting from any of its vertex nodes. Potential $q$ vanishes at all vertex nodes and

$$
\|q\|_{1 / 2+r, \partial K} \leq C\left\|u_{t}-u_{1 t}\right\|_{-1 / 2+r, \partial K}
$$

As the Whitney interpolant depends continuously upon the tangential component $u_{t}$ itself, and it lives in a finite dimensional space, by the standard finite dimensionality 
argument we conclude that the norm of potential $q$ is controlled by the norm of $\mathbf{u}$ alone

$$
\begin{aligned}
\|q\|_{1 / 2+r, \partial K} & \leq C\left\|u_{t}\right\|_{-1 / 2+r, \partial K} \\
& \leq C\left(\|\mathbf{u}\|_{r, K}+\|\operatorname{curl} \mathbf{u}\|_{0, K}\right) .
\end{aligned}
$$

We extend then potential $q$ to the rest of the element using a harmonic (minimum energy) extension. Consequently,

$$
\|q\|_{1+r, K} \leq\left(\|\mathbf{u}\|_{r, K}+\|\operatorname{curl} \mathbf{u}\|_{0, K}\right) .
$$

- $\mathbf{u}_{3}$ is the residual bubble function: $\mathbf{n} \times \mathbf{u}_{3}=0$ on the boundary $\partial K$, and

$$
\operatorname{curl} \mathbf{u}_{3}=\operatorname{curl}\left(\mathbf{u}-\mathbf{u}_{1}\right), \quad \operatorname{div} \mathbf{u}_{3}=\operatorname{div}\left(\mathbf{u}-\mathbf{u}_{1}\right)=0 .
$$

It follows that $\mathbf{u}_{3} \in \mathbf{H}^{1}(K)$ and

$$
\left\|\mathbf{u}_{3}\right\|_{1, K} \leq C\left\|\operatorname{curl}\left(\mathbf{u}-\mathbf{u}_{1}\right)\right\|_{0, K} \leq C\|\operatorname{curl} \mathbf{u}\|_{0, K} .
$$

We use a similar decomposition for the projection-based interpolant $\Pi_{K}^{\text {curl }} \mathbf{u}$ of $\mathbf{u}$,

$$
\Pi_{K}^{\text {curl }} \mathbf{u}=\mathbf{u}_{1}+\operatorname{grad} q_{p}+\mathbf{u}_{p, 3},
$$

with the same Whitney interpolant $\mathbf{u}_{1}$ and $q_{p}=\Pi_{K} q$. Thus $q_{p}$ is only a discrete harmonic function, and $\mathbf{u}_{p, 3}$ is only discrete divergence-free. Obviously,

$$
\mathbf{u}-\Pi_{K}^{\text {curl }} \mathbf{u}=\operatorname{grad}\left(q-q_{p}\right)+\mathbf{u}_{3}-\mathbf{u}_{p, 3} .
$$

The first term admits then the estimate (see [24])

$$
\begin{aligned}
\left\|\operatorname{grad}\left(q-q_{p}\right)\right\|_{0, K} & \leq C p^{-(r-\varepsilon)}\|q\|_{1+r, K} \\
& \leq C p^{-(r-\varepsilon)}\left(\|\mathbf{u}\|_{r, K}+\|\operatorname{curl} \mathbf{u}\|_{0, K}\right) .
\end{aligned}
$$

The estimate of the second term is made possible by Theorem 7: there holds

$$
\left\|\mathbf{u}_{3}-\mathbf{u}_{3, p}\right\|_{0, K} \leq C p^{1 / 2} \inf _{\mathbf{F}_{3, p} \in \mathbf{N}_{\mathrm{N}}^{p}}\left\|\mathbf{u}_{3}-\mathbf{F}_{3, p}\right\|_{0, K}
$$

The approximation result (28) then gives

$$
\begin{aligned}
\left\|\mathbf{u}_{3}-\mathbf{u}_{3, p}\right\|_{0, K} & \leq C p^{-1 / 2}\left\|\mathbf{u}_{3}\right\|_{1, K} \\
& \leq C p^{-1 / 2}\left(\|\mathbf{u}\|_{r, K}+\|\operatorname{curl} \mathbf{u}\|_{0, K}\right) .
\end{aligned}
$$

Combining (30) and (31), we get the final estimate for the master element,

$$
\left\|\mathbf{u}-\Pi_{K}^{\text {curl }} \mathbf{u}\right\|_{0, K} \leq C p^{-(r-\varepsilon)}\left(\|\mathbf{u}\|_{r, K}+\|\operatorname{curl} \mathbf{u}\|_{0, K}\right) .
$$

Step 2: scaling argument. Let $K$ be an arbitrary (unconstrained) square element and let

$$
\Sigma=\hat{K} \ni \boldsymbol{\xi} \rightarrow \mathbf{x} \in K
$$


be the homothetic transformation from the master element $\Sigma$ onto $K$. Recalling the transformation for $\mathbf{H}$ (curl)-conforming elements,

$$
\hat{\mathbf{u}}(\boldsymbol{\xi})=\mathbf{u}(\mathbf{x}) h,
$$

where $h=h_{K}$ is the element size, we follow the standard scaling argument and Step 1 result, to obtain,

$$
\begin{aligned}
\left\|\mathbf{u}-\Pi_{K}^{\text {curl }} \mathbf{u}\right\|_{0, K} & =\left\|\hat{\mathbf{u}}-\Pi^{\text {curl }} \hat{\mathbf{u}}\right\|_{0, \Sigma} \\
& \leq C p^{-(r-\varepsilon)}\left(\|\hat{\mathbf{u}}\|_{r, \Sigma}+\|\operatorname{curl} \hat{\mathbf{u}}\|_{0, \Sigma}\right) .
\end{aligned}
$$

However, the (projection-based) interpolation reproduces polynomials and, by the BrambleHilbert argument and standard interpolation arguments, we get

$$
\begin{aligned}
\left\|\mathbf{u}-\mathbf{u}_{p}\right\|_{0, K} & \leq C p^{-(r-\varepsilon)}\left(|\hat{\mathbf{u}}|_{r, \Sigma}+\|\operatorname{curl} \hat{\mathbf{u}}\|_{0, \Sigma}\right) \\
& \leq C\left(\frac{h}{p}\right)^{(r-\varepsilon)}\left(|\mathbf{u}|_{r, K}+\|\operatorname{curl} \mathbf{u}\|_{0, K}\right) .
\end{aligned}
$$

This finishes the proof.

We have an analogous but slightly different result for element patches.

Lemma 10. Let $P$ be a patch of two, three or four square elements of same size, forming a rectangle, a L-shaped domain and a square, respectively, cf Section 4.2. Let $p$ denote the minimum order of all elements and edges constituting the patch. Let $h$ denote the size of the elements forming the patch. Let $\mathbf{u} \in \mathbf{H}^{r}(P), 0<r<1 / 2$, curl $\mathbf{u} \in L^{2}(P)$, div $\mathbf{u}=0$. There exist constant $C>0$, independent of the element and function $\mathbf{u}$, and constant $r_{P}$, $0<r_{P}<r$, such that

$$
\left\|\mathbf{u}-\Pi_{P}^{\text {curl }} \mathbf{u}\right\|_{0, P} \leq C\left(\frac{h}{p}\right)^{r_{P}}\left(\|\mathbf{u}\|_{r, P}+\|\operatorname{curl} \mathbf{u}\|_{0, P}\right) .
$$

$B y \Pi_{P}^{\text {curl }} \mathbf{u}$ we understand the projection-based interpolation (22) done on the patch.

Proof. The reasoning follows the same lines as for the preceding lemma. We revisit the main steps and point out to differences.

- $\mathbf{u}_{1}$ plays on the patch $P$ a similar (but weaker) role as the lowest degree Whitney interpolant on $K: \mathbf{u}_{1} \in \mathbf{N}^{1 \mid 0}(P)$, div $\mathbf{u}_{1}=0$ in $P$ and $\mathbf{u}_{1}$ compensates for the mean value of the tangential trace of $\mathbf{u}$ on the whole boundary $\partial P$ :

$$
\int_{\partial P} \mathbf{n} \times\left(\mathbf{u}-\mathbf{u}_{1}\right) d s=0 .
$$

For $\mathbf{u}_{1}$ we may take a field of the form $\left.\gamma \mathbf{e}\right|_{P}$ where $\mathbf{e}$ is any non-zero element of $\mathbf{N}^{1 \mid 0}(\hat{P})$ on the convex hull $\hat{P}$ of $P$, and $\gamma$ is a suitable constant. 
- The potential $q$ is still obtained by first integrating $u_{t}-u_{1 t}$ along $\partial P$. Now it does not vanish at the corners, but we still have $q \in H^{1 / 2+r}(\partial P)$, so we can take its harmonic extension in $P$ to find $q \in H^{1+r}(P)$.

- We still have a decomposition like $(29) \mathbf{u}=\mathbf{u}_{1}+\operatorname{grad} q+\mathbf{u}_{3}$ with a divergence free patch bubble function $\mathbf{u}_{3}$. But for the L-shaped patches, $\mathbf{u}_{3}$ is no longer an $\mathbf{H}^{1}$ function, however $\mathbf{u}_{3}$ belongs to $\mathbf{H}^{1 / 2+r_{P}}(P)$, with $r_{P}>0$ (here $r_{P}$ is any constant $\left.<\frac{1}{6}\right),[18]$.

- At the discrete level, we have $\Pi_{P}^{\text {curl }} \mathbf{u}=\mathbf{u}_{1}+\operatorname{grad}\left(\Pi_{P} q\right)+\mathbf{u}_{3, p}$. The estimate corresponding to (30), of $\left\|\operatorname{grad}\left(q-\Pi_{P} q\right)\right\|_{0, P}$ does not follow directly from element estimates but it can be obtained extending arguments from [24]. Alternatively, the $H^{1}$ patch interpolant $\Pi_{P} q$ can be seen as the Galerkin approximation to the solution of Laplace equation on the patch, with Dirichlet boundary conditions and right approximation of Dirichlet data (in $H^{1 / 2}$ norm). The corresponding estimates can be found in [43].

- The bound on $\left\|\mathbf{u}_{3}-\mathbf{u}_{3, p}\right\|_{0, P}$ corresponding to (31) does not follow directly from the $L^{2}$ - stability result for a single element. Instead, we proceed by comparing the patch interpolant $\mathbf{u}_{3, p}=\Pi_{P}^{\text {curl }} \mathbf{u}_{3}$ with the union of interpolants $\Pi_{K}^{\text {curl }} \mathbf{u}_{3}$ corresponding to elements $K$ contributing to the patch, denoted by $\mathbf{v}_{3, p}$ :

$$
\left.\mathbf{v}_{3, p}\right|_{K}=\Pi_{K}^{\text {curl }} \mathbf{u}_{3}, \quad \forall K \subset P .
$$

Both operators $\Pi_{P}^{\text {curl }}$ and $\left(\Pi_{K}^{\text {curl }}\right)_{K \subset P}$, acting from $\mathbf{H}_{0}(\operatorname{curl}, P) \cap \mathbf{H}^{\varepsilon}(P)$, satisfy the commutativity property for the de Rham diagram. The $L^{2}$-projections of curl $\mathbf{u}$ done on the whole patch or elementwise, are identical. Consequently,

$$
\operatorname{curl} \mathbf{u}_{3, p}=\operatorname{curl} \mathbf{v}_{3, p},
$$

and the two functions may differ only by a gradient of potential $\phi$ that is zero on the patch boundary $\partial P$ and lives in the patch $\mathrm{FE}$ space. It follows from the fact that $\mathbf{u}_{3, p}$ is discrete divergence-free that

$$
\begin{aligned}
\left\|\mathbf{u}_{3}-\mathbf{u}_{3, p}\right\|_{0, P} & \leq \inf _{\phi}\left\|\mathbf{u}_{3}-\mathbf{u}_{3, p}-\operatorname{grad} \phi\right\|_{0, P} \\
& \leq\left\|\mathbf{u}_{3}-\mathbf{v}_{3, p}\right\|_{0, P} .
\end{aligned}
$$

Coming back to the definition of $\mathbf{v}_{3, p}$, we finally obtain

$$
\left\|\mathbf{u}_{3}-\mathbf{u}_{3, p}\right\|_{0, P} \leq \sum_{K \subset P}\left\|\mathbf{u}_{3}-\Pi_{K}^{\mathrm{curl}} \mathbf{u}_{3}\right\|_{0, P}
$$

The estimation can now be done elementwise on each unconstrained element $K \subset P$ utilizing Lemma 9 for $\mathbf{u}:=\left.\mathbf{u}_{3}\right|_{K}$, noting that $\left.\operatorname{div} \mathbf{u}_{3}\right|_{K}=0$.

We are ready now to formulate and prove our final result. 
Theorem 11. Starting with a regular mesh on $\Omega$ we perform consecutive hp-refinements, enforcing the 1-irregularity and minimum rules, constructing meshes $\mathfrak{M}_{h p}$. We assume that

$$
\max _{K \in \mathfrak{M}_{h p}} \frac{h_{K}}{p_{K}} \rightarrow 0 .
$$

Let $\mathbf{u}_{h p} \in \mathbf{X}_{h p}$ be an arbitrary sequence of FE functions on $\mathfrak{M}_{h p}$, such that $\mathbf{u}_{h p} \times \mathbf{n}=0$ on $\partial \Omega$. We assume that the functions $\mathbf{u}_{h p}$ are discrete divergence free, i.e.

$$
\left(\mathbf{u}_{h p}, \operatorname{grad} \phi_{h p}\right)=0, \quad \forall \phi_{h p} \in \mathbb{Q}_{h p}
$$

We also assume that the $\mathbf{u}_{h p}$ are uniformly bounded in the space $\mathbf{H}(\mathrm{curl}, \Omega)$

$$
\left\|\operatorname{curl} \mathbf{u}_{h p}\right\| \leq 1 \text {. }
$$

Then there exists a subsequence $\mathbf{u}_{h p}$, (denoted with the same symbol) converging strongly in $L^{2}(\Omega)$ to a limit $^{2} \mathbf{u}$

$$
\left\|\mathbf{u}_{h p}-\mathbf{u}\right\| \rightarrow 0
$$

Proof. Step 1. Following Kikuchi's reasoning (see [35]), we introduce a sequence of divergence-free functions $\mathbf{u}^{h p}$, satisfying the same essential boundary conditions, such that

$$
\operatorname{curl} \mathbf{u}^{h p}=\operatorname{curl} \mathbf{u}_{h p}, \quad\left(\mathbf{u}^{h p}, \operatorname{grad} \phi\right)=0 \quad \forall \phi \in H_{0}^{1}(\Omega) .
$$

We have

$$
\mathbf{u}_{h p}=\mathbf{u}^{h p}+\operatorname{grad} q^{h p}
$$

where $q^{h p}$ is solution to

$$
\begin{aligned}
& q^{h p} \in H_{0}^{1}(\Omega) \\
& \left(\operatorname{grad} q^{h p}, \operatorname{grad} \phi\right)=\left(\mathbf{u}_{h p}, \operatorname{grad} \phi\right), \quad \forall \phi \in H_{0}^{1}(\Omega) .
\end{aligned}
$$

It follows from the regularity results of [18] that

$$
\mathbf{u}^{h p} \in \mathbf{H}^{r}(\Omega), r>0,
$$

with a uniform bound on the $\mathbf{H}^{r}$ norm,

$$
\left\|\mathbf{u}^{h p}\right\|_{\mathbf{H}^{r}(\Omega)} \leq C .
$$

By a standard compactness argument, there exists a subsequence $\mathbf{u}^{h p}$ converging strongly in $L^{2}(\Omega)$ to a limit $\mathbf{u}$. We are going to prove that $\operatorname{grad} q^{h p} \rightarrow 0$, and obtain consequently that $\mathbf{u}_{h p}$ converges to the same limit $\mathbf{u}$.

Step 2. Applying the interpolation operator to both sides of the equation, and using the commutativity of interpolation and the fact that the interpolation preserves FE spaces, we get

$$
\mathbf{u}_{h p}=\Pi^{\mathrm{curl}} \mathbf{u}^{h p}+\operatorname{grad} \Pi q^{h p} .
$$

\footnotetext{
${ }^{2}$ Notice that limit satisfies $\|\operatorname{curl} \mathbf{u}\| \leq 1$, and that $\mathbf{u}$ is divergence-free.
} 
Subtracting (34) from (33) we get

$$
-\operatorname{grad}\left(q^{h p}-\Pi q^{h p}\right)=\mathbf{u}^{h p}-\Pi^{\mathrm{curl}} \mathbf{u}^{h p}
$$

It follows from (33) that $\operatorname{grad} q^{h p}$ is orthogonal to all discrete gradients. Consequently,

$$
\left\|\operatorname{grad} q^{h p}\right\|=\inf _{q_{h p} \in Q_{h p}}\left\|\operatorname{grad}\left(q^{h p}-q_{h p}\right)\right\| \leq\left\|\operatorname{grad}\left(q^{h p}-\Pi q^{h p}\right)\right\|=\left\|\mathbf{u}^{h p}-\Pi^{\operatorname{curl}} \mathbf{u}^{h p}\right\|
$$

It is sufficient, therefore, to prove that the interpolation error of functions $\mathbf{u}^{h p}$ converges uniformly to zero.

Step 3. Applying Lemmas 9 and 10, we obtain

$$
\begin{aligned}
\left\|\mathbf{u}^{h p}-\Pi^{\mathrm{curl}} \mathbf{u}^{h p}\right\|_{0, \Omega}^{2} & =\sum_{P}\left\|\mathbf{u}^{h p}-\Pi^{\mathrm{curl}} \mathbf{u}^{h p}\right\|_{0, P}^{2} \\
& \leq C \sum_{P}\left(\frac{h_{P}}{p_{P}}\right)^{2 r_{P}}\left(\left\|\mathbf{u}^{h p}\right\|_{r, P}+\left\|\operatorname{curl} \mathbf{u}^{h p}\right\|_{0, P}\right)^{2}
\end{aligned}
$$

Here $r$ is the global regularity constant and $r_{P}<r$ denote the patch constants discussed in Lemma 10 (we also consider unconstrained elements $K$ as one-element patches and, applying Lemma 9, take $r_{P}$ as any number between 0 and $r$ in this case) As $r_{P}$ depends only upon the shape of the patch and the number of different patches is finite, the $L^{2}$ interpolation error must converge to zero, if the maximum ratio of patch size and (minimum) order converges to zero,

$$
\max _{P} \frac{h_{P}}{p_{P}} \rightarrow 0 .
$$

Notice, finally, that the 1-irregularity and max rules imply that the last condition follows from assumption (32).

Remark 5. Examining our proof, we see that we have proved the following property: There exists a sequence $\delta_{h p}$ converging to 0 such that

$$
\begin{aligned}
& \forall \mathbf{u}_{h p} \in \mathbf{X}_{h p}, \quad \text { discrete divergence free, } \\
& \quad \exists \mathbf{u}^{h p} \in \mathbf{H}_{0}(\operatorname{curl}, \Omega) \text { with } \operatorname{div} \mathbf{u}^{h p}=0:\left\|\mathbf{u}^{h p}-\mathbf{u}_{h p}\right\| \leq C \delta_{h p}\left(\left\|\mathbf{u}_{h p}\right\|+\left\|\operatorname{curl} \mathbf{u}_{h p}\right\|\right) .
\end{aligned}
$$

Here $C>0$ does not depend on $\mathbf{u}_{h p}$. Condition (35) implies the discrete compactness property, cf. [6, 7]. It also implies the quasi-optimality of the discrete electric Maxwell problems for any fixed frequency which is not an eigenfrequency of the continuous problem, see $[27,10,15]$. 


\section{Conclusions}

Relying on our main result Theorem 11 and on [16], we can conclude the convergence of eigenvalue approximation along the following lines.

Let $\Omega$ be a simply connected polygonal domain with sides parallel to the coordinate axes. We consider the Maxwell eigenvalue problem on $\Omega$ :

$$
\begin{aligned}
& \text { Find } \mathbf{u} \in \mathbf{H}(\operatorname{curl}, \Omega), \mathbf{u} \neq 0, \text { and } \lambda \neq 0: \\
& \qquad \int_{\Omega} \operatorname{curl} \mathbf{u} \operatorname{curl} \mathbf{v} d \mathbf{x}=\lambda \int_{\Omega} \mathbf{u} \cdot \mathbf{v} d \mathbf{x}, \quad \forall \mathbf{v} \in \mathbf{H}(\operatorname{curl}, \Omega) .
\end{aligned}
$$

The condition $\lambda \neq 0$ implies that eigenvectors $\mathbf{u}$ are divergence free. The converse is also true since $\Omega$ is simply connected. The eigenvalues $\lambda$ have a finite multiplicity and form an increasing sequence of positive numbers without accumulation point. We denote by $\lambda^{1} \leq \lambda^{2} \leq \cdots \leq \lambda^{k} \leq \cdots$ the sequence of eigenvalues with repetition according to their multiplicities.

We choose a sequence of $h p$ finite element spaces $\mathbf{X}_{h p}$ satisfying the assumptions of Theorem 11 and define the approximated problems by.

$$
\begin{aligned}
& \text { Find } \mathbf{u}_{h p} \in \mathbf{X}_{h p}, \mathbf{u}_{h p} \neq 0 \text {, and } \lambda_{h p} \neq 0 \text { : } \\
& \qquad \int_{\Omega} \operatorname{curl} \mathbf{u}_{h p} \operatorname{curl} \mathbf{v}_{h p} d \mathbf{x}=\lambda_{h p} \int_{\Omega} \mathbf{u}_{h p} \cdot \mathbf{v}_{h p} d \mathbf{x}, \quad \forall \mathbf{v}_{h p} \in \mathbf{X}_{h p} .
\end{aligned}
$$

The discrete eigenvectors $\mathbf{u}_{h p}$ are discrete divergence free. We denote by $\lambda_{h p}^{1} \leq \lambda_{h p}^{2} \leq \cdots \leq$ $\lambda_{h p}^{k} \leq \cdots$ the sequence of eigenvalues with repetition according to their multiplicities.

Assumption (32) guarantees that the conditions (CAS) (approximation in $\mathbf{H}(\operatorname{curl}, \Omega)$ ) and $(\mathrm{CDK})$ (approximation in the kernel of the curl operator) of [16] are satisfied. Theorem 11 yields condition (DCP) of discrete compactness. Thus [16, Theorem 6.9] yields that the sequence of problems (37) is a spurious-free spectrally correct approximation of problem (36). As a consequence, we have

$$
\forall k \geq 1, \quad \lambda_{h p}^{k} \longrightarrow \lambda^{k} \quad \text { as } \quad \max _{K \in \mathfrak{M}_{h p}} \frac{h_{K}}{p_{K}} \rightarrow 0 .
$$

Let us recall that the spectral correctness alone would provide a weaker statement, according to which the correct numbering of discrete eigenvalues which ensures (38) should be done by discarding small eigenvalues (and not only zero eigenvalues). "Small" means that the maximal size $\varepsilon_{h p}$ of the discarded ones tends to zero as $h_{K} / p_{K}$ tends to zero. The consequence of the spurious free property is that $\varepsilon_{h p}$ is equal to zero.

According to [16, Theorem 6.11] the three conditions (CAS), (CDK) and (DCP) imply condition $(\mathrm{CHN})$ too. Condition $(\mathrm{CHN})$ is the one which allows the application of the theory 
of [28], see also [16, Remark 4.11]. This implies, for example, that if $\lambda^{k}$ is a simple eigenvalue, there holds the estimate

$$
\left|\lambda^{k}-\lambda_{h p}^{k}\right| \leq C_{k}\left(\min _{\mathbf{v}_{h p} \in \mathbf{X}_{h p}}\left\|\mathbf{u}^{k}-\mathbf{v}_{h p}\right\|_{\mathbf{H}(\operatorname{curl}, \Omega)}\right)^{2} .
$$

Here $\mathbf{u}^{k}$ is a normalized eigenvector associated with $\lambda^{k}$. Less sharp estimates can be deduced by this argument in the case of multiple eigenvalues.

In $[21,22]$, it is proved that the regularity of the eigenvectors $\mathbf{u}^{k}$ can be described in terms of weighted analytic spaces (close to the countably normed spaced of [4]), via a decomposition $\nabla \varphi^{k}+\mathbf{w}^{k}$ where the potential $\varphi^{k}$ concentrates the strongest singularities. Combining this with the approximation result proved in [1] for Raviart-Thomas elements, it is possible to deduce an exponential estimate in our case

$$
\left|\lambda^{k}-\lambda_{h p}^{k}\right| \leq C_{k} e^{-b_{k} N^{1 / 3}}, \quad b_{k}>0 .
$$

Here $N$ is the dimension of $\mathbf{X}_{h p}$. Note that $N^{1 / 3}$ is a $\mathcal{O}(p)$.

Our final comment concerns the validity of our result for meshes obtained using the so called algebraic mesh generators. This is the case when the actual physical domain is partitioned into a finite number of (possibly curvilinear) quadrilaterals, each of them being the image of a reference unit square through a smooth map. The maps are compatible in the sense that parametrizations for two quadrilaterals adjacent to a common edge, provide an identical parametrization for the edge. The original Maxwell problem can then be restated on a collection of reference square domains with appropriate interface conditions, and modified material properties resulting from the change of coordinates. According to the result of Caorsi et al [16], the discrete compactness property for constant material data implies the corresponding discrete compactness property for the case of general, possibly anisotropic, material data. As the discretization in the original domain with parametric, exact geometry elements, is equivalent with the discretization of the modified problem on the reference squares using square elements discussed in this paper, our analysis applies to such a case as well. We emphasize that the situation is essentially different when unstructured mesh generators are used, and the geometry of individual quadrilateral elements is no longer controlled by global (and sufficiently smooth) maps, compare [3].

\section{REFERENCES}

1. M. Ainsworth and K. Pinchedez, hp-approximation theory for BDFM and RT finite elements on quadrilaterals, SIAM J. Numer. Anal. 40 (2002), no. 6, 2047-2068.

2. D. N. Arnold, Differential complexes and numerical stability, Proceedings of the International Congress of Mathematicians, Vol. I (Beijing, 2002) (Beijing), Higher Ed. Press, 2002, pp. 137-157. MR MR1989182 (2004h:65115)

3. D. N. Arnold, D. Boffi, and R. S. Falk, Quadrilateral H(div) finite elements, SIAM J. Numer. Anal. (2004), to appear. 
4. I. Babuška and B. Q. Guo, Regularity of the solution of elliptic problems with piecewise analytic data. I. Boundary value problems for linear elliptic equation of second order, SIAM J. Math. Anal. 19 (1988), no. 1, 172-203.

5. I. Babuška and J. Osborn, Eigenvalue problems, Handbook of numerical analysis, Vol. II, Handb. Numer. Anal., II, North-Holland, Amsterdam, 1991, pp. 641-787. MR MR1115240

6. D. Boffi, Fortin operator and discrete compactness for edge elements, Numer. Math. 87 (2000), no. 2, 229-246.

7. (2001), no. 1, 33-38.

8. D. Boffi, L. Demkowicz, and M. Costabel, Discrete compactness for $p$ and hp $2 D$ edge finite elements, Math. Models Methods Appl. Sci. 13 (2003), no. 11, 1673-1687. MR 2024467

9. D. Boffi, P. Fernandes, L. Gastaldi, and I. Perugia, Computational models of electromagnetic resonators: analysis of edge element approximation, SIAM J. Numer. Anal. 36 (1999), no. 4, 1264-1290.

10. D. Boffi and L. Gastaldi, Edge finite elements for the approximation of Maxwell resolvent operator, M2AN Math. Model. Numer. Anal. 36 (2002), no. 2, 293-305.

11. D. Boffi, F. Kikuchi, and J. Schöberl, Edge element computation of Maxwell's eigenvalues on general quadrilateral meshes, Math. Mod. Meth. Appl. Sci. (2005), to appear.

12. A. Bossavit, Mixed finite elements and the complex of Whitney forms, The mathematics of finite elements and applications, VI (Uxbridge, 1987), Academic Press, London, 1988, pp. 137-144. MR MR956893 (89k:58028)

13. __ Un nouveau point de vue sur les éléments finis mixtes, Matapli (Bulletin de la Société de Mathématiques Appliquëes et Industrielles (1989), 23-35.

14. A. Buffa, M. Costabel, and M. Dauge, Algebraic convergence for anisotropic edge elements in polyhedral domains, Preprint, Pavia N. 28-PV, Istituto di Matematica Applicata e Tecnologie Informatiche CNR, 2003. To appear in Numer. Math.

15. A. Buffa, R. Hiptmair, T. von Petersdorff, and C. Schwab, Boundary element methods for Maxwell transmission problems in Lipschitz domains, Numer. Math. 95 (2003), no. 3, 459-485.

16. S. Caorsi, P. Fernandes, and M. Raffetto, On the convergence of Galerkin finite element approximations of electromagnetic eigenproblems, SIAM J. Numer. Anal. 38 (2000), no. 2, 580-607 (electronic).

17. F. Chatelin, Spectral approximation of linear operators, Academic Press, Inc., 1983.

18. M. Costabel and M. Dauge, Singularities of electromagnetic fields in polyhedral domains, Arch. Ration. Mech. Anal. 151 (2000), no. 3, 221-276.

19. _ Computation of resonance frequencies for Maxwell equations in non-smooth domains, Topics in Computational Wave Propagation, Lect. Notes Comput. Sci. Eng., vol. 31, Springer, Berlin, 2003, pp. $125-161$.

20. M. Costabel, M. Dauge, and S. Nicaise, Singularities of Maxwell interface problems, M2AN Math. Model. Numer. Anal. 33 (1999), no. 3, 627-649.

21. M. Costabel, M. Dauge, and C. Schwab, Exponential convergence of hp-FEM for Maxwell's equations with weighted regularization in polygonal domains, Math. Models Methods Appl. Sci. 15 (2005), no. 4, 575-622.

22. M. Costabel, M. Dauge, and C. Schwab, Exponential convergence of the weighted regularization method for maxwell eigenvalue problems, Proceedings of International Conference on Electromagnetics in Advanced Applications (ICEAA), Torino, September 12-16, 2005, 2005.

23. L. Demkowicz, Fully automatic hp-adaptivity for Maxwell's equations, Tech. Report 03-45, ICES, 2003.

24. L. Demkowicz and I. Babuška, $p$ interpolation error estimates for edge finite elements of variable order in two dimensions, SIAM J. Numer. Anal. 41 (2003), no. 4, 1195-1208 (electronic). 
25. L. Demkowicz and A. Buffa, $H^{1}, H($ curl $)$ and $H($ div $)$-conforming projection-based interpolation in three dimensions, Tech. Report 04-24, ICES, 2004.

26. L. Demkowicz, P. Monk, L. Vardapetyan, and W. Rachowicz, de Rham diagram for hp finite element spaces, Comput. Math. Appl. 39 (2000), no. 7-8, 29-38.

27. L. Demkowicz and L. Vardapetyan, Modeling of electromagnetic absorption/scattering problems using hpadaptive finite elements, Comput. Methods Appl. Mech. Engrg. 152 (1998), no. 1-2, 103-124, Symposium on Advances in Computational Mechanics, Vol. 5 (Austin, TX, 1997).

28. J. Descloux, N. Nassif, and J. Rappaz, On spectral approximation. I. The problem of convergence, RAIRO Anal. Numér. 12 (1978), no. 2, 97-112, iii.

29. B. M. Dillon, P. T. S. Liu, and J. P. Webb, Spurious modes in quadrilateral and triangular edge elements, COMPEL 13, Suppl. A (1994), 311-316.

30. P. Fernandes and M. Raffetto, Counterexamples to the currently accepted explanation for spurious modes and necessary and sufficient conditions to avoid them, IEEE Trans. on Magnetics 38 (2002), no. 2, 653656.

31. F. Gardini, Discrete compactness property for quadrilateral finite element spaces, Numer. Methods Partial Differential Equations 21 (2005), no. 1, 41-56. MR MR2100299

32. P. Grisvard, Singularities in boundary value problems, Recherches en Mathématiques Appliquées [Research in Applied Mathematics], vol. 22, Masson, Paris, 1992.

33. R. Hiptmair, Finite elements in computational electromagnetism, Acta Numer. 11 (2002), 237-339.

34. J. Douglas Jr. and J.E. Roberts, Mixed finite element methods for second order elliptic problems, Mat. Apl. Comput. 1 (1982), 91-103.

35. F. Kikuchi, On a discrete compactness property for the Nédélec finite elements, J. Fac. Sci. Univ. Tokyo Sect. IA Math. 36 (1989), no. 3, 479-490.

36. W. McLean, Strongly elliptic systems and boundary integral equations, Cambridge University Press, 2000.

37. P. Monk, On the p- and hp-extension of Nédélec's curl-conforming elements, J. Comput. Appl. Math. 53 (1994), no. 1, 117-137.

38. _ Finite element methods for Maxwell's equations, Numerical Mathematics and Scientific Computation, Oxford University Press, 2003.

39. P. Monk and L. Demkowicz, Discrete compactness and the approximation of Maxwell's equations in $\mathbb{R}^{3}$, Mathematics of Computation $\mathbf{7 0}$ (2000), no. 234, 507-523.

40. J.-C. Nédélec, Mixed finite elements in $\mathbf{R}^{3}$, Numer. Math. 35 (1980), no. 3, 315-341.

41. _ A new family of mixed finite elements in $\mathbb{R}^{3}$, Numer. Math. 50 (1986), no. 1, 57-81.

42. W. Rachowicz and L. Demkowicz, An hp-adaptive finite element method for electromagnetics. part I. Data structure and constrained approximation, Comput. Methods Appl. Mech. Engrg. 187 (2000), no. 12, 307-337.

43. Ch. Schwab, $p$ - and hp-finite element methods, Numerical Mathematics and Scientific Computation, The Clarendon Press Oxford University Press, New York, 1998, Theory and applications in solid and fluid mechanics. 
Dipartimento di Matematica "F. Casorati", Università di Pavia, I-27100 Pavia, Italy

E-mail address: daniele.boffi@unipv.it

$U R L$ : http://www-dimat.unipv.it/boffi/

IRMaR, Institut Mathématique, Université de Rennes 1, 35042 Rennes, France

E-mail address: costabel@univ-rennes1.fr

$U R L$ : http://perso.univ-rennes1.fr/martin.costabel/

IRMaR, Institut Mathématique, Université de Rennes 1, 35042 Rennes, France

E-mail address: dauge@univ-rennes1.fr

URL: http://perso.univ-rennes1.fr/monique.dauge/

The Institute for Computational Engineering and Sciences, The University of Texas at Austin, Austin, TX 78712, USA

E-mail address: leszek@ices.utexas.edu

URL: http://www.ices.utexas.edu/ leszek/ 